\title{
Review
}

\section{Signals Fly when Kinases Meet RHO-OF-PLANTS (ROP) Small G-Proteins}

Attila Fehér ${ }^{*}$, Dézi Bianka Lajkó

Institute of Plant Biology, Biological Research Centre, Hungarian Academy of Sciences, Temesvári krt. 62., H-6726 Szeged, Hungary

\footnotetext{
*Author for correspondence:
}

Attila Fehér, Institute of Plant Biology, Biological Research Centre, Hungarian Academy of Sciences, P.O. Box 521, H-6701 Szeged, Hungary

E-mail: feher.attila@brc.mta.hu 


\begin{abstract}
Rho-type small GTP-binding plant proteins function as two-state molecular switches in cellular signalling. There is accumulating evidence that RHO-OF-PLANTS (ROP) signalling is positively controlled by plant receptor kinases, through the ROP GUANINE NUCLEOTIDE EXCHANGE FACTOR proteins. These signalling modules regulate cell polarity, cell shape, hormone responses, and pathogen defence, among other things. Other ROP-regulatory proteins might also be subjected to protein phosphorylation by cellular kinases (e.g., MITOGEN-ACTIVATED PROTEIN KINASEs or CALCIUM-DEPENDENT PROTEIN KINASEs), in order to integrate various cellular signalling pathways with ROP GTPase-dependent processes. In contrast to the role of kinases in upstream ROP regulation, much less is known about the potential link between ROP GTPases and downstream kinase signalling. In other eukaryotes, Rho-type G-protein-activated kinases are widespread and play a key role in many cellular processes. Recent data indicate the existence of structurally different ROP-activated kinases in plants, but their ROP-dependent biological functions still need to be validated. In addition to these direct interactions, ROPs may also indirectly control the activity of MITOGEN-ACTIVATED PROTEIN KINASEs or CALCIUM-DEPENDENT PROTEIN KINASEs. These kinases may therefore function as upstream as well as downstream kinases in ROP-mediated signalling pathways, as in the case of the phosphatidylinositol monophosphate kinases involved in cell polarity establishment.
\end{abstract}

\title{
Highlights
}

- Various types of receptor-like kinases positively regulate ROP signalling.

- The receptor kinase-RopGEF-ROP signalling module is used in a wide variety of cellular processes. 
- ROPs and their regulators are likely to be subjected to regulation by protein phosphorylation.

- ROPs might play a role in directly and indirectly activating downstream kinase signalling.

Key words: mitogen-activated protein kinase; protein phosphorylation; ROP GTPase; guanine nucleotide exchange factor; ROP-binding kinase; receptor-like kinase

\section{Contents}

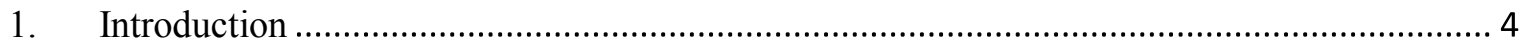

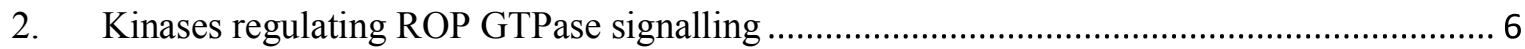

2.1 The CLAVATA1 receptor kinase complex …….................................................................. 7

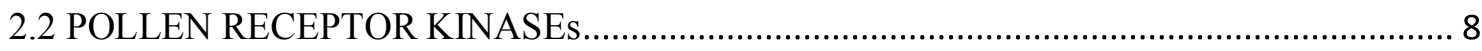

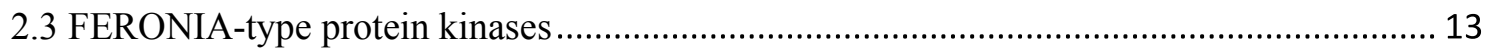

2.4 The TRANSMEMBRANE KINASE receptor kinases .................................................... 19

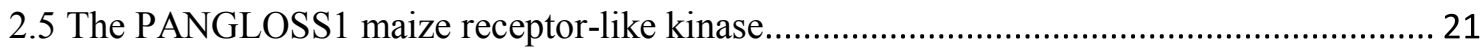

2.6 The LYSIN MOTIF DOMAIN RECEPTOR-LIKE KINASEs ......................................... 23

2.7 Further RopGEF-ROP signalling modules potentially linked to receptor kinases ............... 24

2.8 Non-receptor protein kinases upstream of ROP GTPase signalling ...................................... 25

3. Protein kinases regulated by ROP GTPase signalling ........................................................ 29

3.1 The ROP-activated RECEPTOR-LIKE CYTOPLASMIC KINASEs................................... 30

3.2 The SNF1-RELATED PROTEIN KINASE TYPE 2 kinase is indirectly regulated by ROPs

3.3 ROP GTPases and the MITOGEN-ACTIVATED PROTEIN KINASE cascades ................ 34

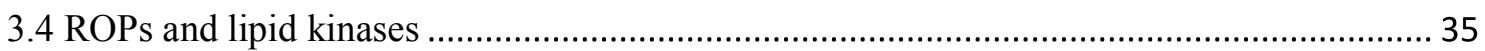

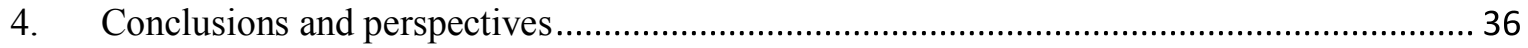

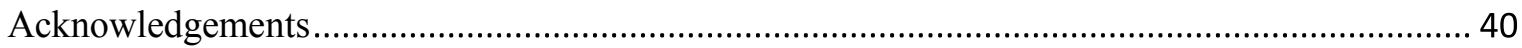

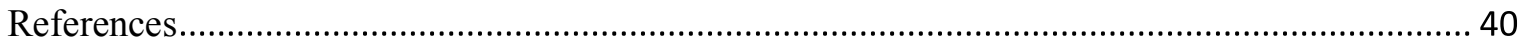




\section{Introduction}

Rho-type small GTP-binding (or G-) proteins are among the key cellular signalling molecules in all eukaryotes (see, e.g., [1]). Their regulatory role is based on their capability to bind and hydrolyse GTP, and switch between two states: the active GTP- and the inactive GDP-bound conformations (Fig. 1). The GTP-bearing Rho G-proteins perform their regulatory function through a conformation-specific interaction with target (effector) proteins ([1]; Fig. 1). The power of G-proteins to alternate between active and inactive conformations allows these proteins to serve as ideal molecular switches for the transmission of discrete "ON-OFF" signals within the cells (Fig. 1). A number of regulatory steps control small Gproteins and fine-tune their signalling capacity (see, e.g., [2-4]; Fig. 1). G-protein activation is primarily achieved via the GDP-to-GTP exchange facilitated by guanine nucleotide exchange factors (GEFs). The inactivation of GTPases is the result of their intrinsic GTP-hydrolysing activity, stimulated by GTPase-activating proteins. Guanine nucleotide dissociation inhibitors (GDIs) regulate the membrane association and block the spontaneous activation of small GTPases. A further important biochemical feature of Rho proteins is their post-translational modification by lipids, determining their specific interaction with membrane microdomains.

Variations in the expression pattern, structure, and post-translational modification of Rho proteins influence their subcellular locations as well as their interactions with a number of upstream regulator and downstream effector molecules. These spatially and temporally regulated signalling relations allow these small GTPases to function as sophisticated modulators of a remarkably complex and diverse range of cellular processes. Rho G-proteins are primarily considered to play roles in the regulation of cytoskeletal functions, gene transcription, and the activity of plasma membrane NADPH oxidase [1-3].

Plants have a sole subfamily of Rho-type GTPases, called ROPs (Rho of plants), the members of which are structurally distinct from the proteins in the Rho, Rac, and Cdc42 
subfamilies of other eukaryotes [2, 3] (Table 1). The Rop subfamily is represented by 14 proteins in Arabidopsis and by seven in rice [5]. Some of the molecular mechanisms allowing ROPs to receive and transmit signals are conserved among yeasts, animals, and plants, whereas others are specific [3] (Table 1).

For example, the receptor tyrosine kinases and G-protein-coupled receptors that are upstream regulators of Rho-type GTPases in animals are most likely missing from plants [6, 7]. However, plants do have a large number of receptor-like serine/threonine kinases [6]. The receptor-dependent activation of animal Rho-type G-proteins is mediated by guanine nucleotide exchange factors (GEFs), which are structurally unrelated in plants (Table 1). GEFs of non-plant Rho GTPases typically contain Dbl homology or DOCK180 catalytic domains, while most known RopGEFs have a specific plant ROP nucleotide exchanger (PRONE) domain with the same biochemical function [2]. The exception is a single DOCK180 domain-containing RopGEF, the SPIKE1 protein, described in Arabidopsis [8]. There is accumulating experimental evidence suggesting that receptor kinases regulate Rho GTPase signalling through GEFs in plants [9] as well as in animals [10], despite the fact that the participating proteins are distinct (Table 1).

It is not only the upstream ROP regulators that have specific features. ROPs share only a few downstream effectors with other eukaryotes (Table 1), such as the plasma membrane NADPH oxidase and certain actin nucleation complexes [3]. ROPs have several plant-specific effectors, including enzymes that play a role in cell wall synthesis, and plant-specific small scaffold proteins [2-4]. One of the most characteristic effector families of animal Rho-type GTPases - the Cdc42/Rac interactive binding motif-containing (CRIB-containing) kinases (e.g., Ste20 and related kinases in yeast, p21-activated kinases in animals; [11]) — is missing from plants (Table 1), and our present knowledge on the link between ROPs and downstream kinase signalling is limited. 
The aim of this review is to give an overview of the existing information about the insertion of ROP GTPases into upstream as well as downstream kinase signalling pathways in plants.

\section{Kinases regulating ROP GTPase signalling}

Signal perception through cell surface receptors is an important feature of all living organisms. The nature and abundance of various receptor configurations are highly divergent among the various taxa. A huge family of receptor serine/threonine kinases (usually referred to as receptor-like kinases), for example, characterizes land plants. The overall structure of these plant kinases (extracellular, transmembrane and cellular kinase domains) is very similar to that of the animal receptor tyrosine kinases, yet plant and animal receptor kinases have an independent evolutionary origin [6]. The abundance of plant receptor-like kinases is combined with the structural diversity underlying their functional divergence: they are involved in a variety of developmental, environmental and hormonal signalling pathways [12]. Due to the importance of this receptor family in plants, there have been considerable efforts to characterize its members, involving the identification of their ligands and downstream targets. Lately, these investigations have demonstrated that several plant receptor-like kinases affect the signalling activity of ROP G-proteins ([9]; see Sections 2.12.7).

In animal cells, receptor tyrosine kinase signalling to Rho proteins is a general signal transduction mechanism: more than half of the 58 known human receptor tyrosine kinases are implicated in Rho activation [10]. Receptor tyrosine kinases activate the Rho proteins indirectly, through the activation of RhoGEFs that facilitate the GDP-to-GTP exchange of Rho G-proteins [10]. Multiple receptor tyrosine kinases may activate the same RhoGEF-Rho signalling pathway, and the same receptor tyrosine kinase can activate multiple 
RhoGEFs/Rho proteins. Therefore, a complex combinatorial network of receptor tyrosine kinases, RhoGEFs and Rho proteins contributes to the specificity of the responses evoked by diverse signals in various tissue and cell types, each expressing a special subset of these proteins [10]. As discussed below in detail, recent findings indicate that similar signalling mechanisms have evolved in plants independently, since although plant receptor kinases, RopGEFs and ROPs are structurally different proteins from their functionally equivalent metazoan counterparts, they are also members of similar signalling modules. These presently recognized plant modules are summarized in Table 2, and are discussed in detail in Sections $2.1-2.7$

\subsection{The CLAVATA1 receptor kinase complex}

The first indication that ROP G-proteins and receptor-like kinases are interlinked came from the characterization of the Arabidopsis CLAVATA1 receptor-like kinase complex [13]. CLAVATA1 is a receptor kinase that regulates the size of the stem cell population in the Arabidopsis shoot meristem. CLAVATA1 is a member of two receptor complexes: one is 185 $\mathrm{kD}$, the other is $450 \mathrm{kD}$ [13]. Only the $450 \mathrm{kD}$ complex, which was hypothesized to include the active receptor, contains a ROP G-protein as determined by co-immunoprecipitation experiments using CLAVATA1 and ROP antibodies. Due to the conserved structure of plant ROPs, the used antibody could not reveal which specific ROP GTPase was present in the complex. It was proposed that the active CLAVATA1 receptor signals, via the ROP protein, towards a MITOGEN-ACTIVATED PROTEIN KINASE (MAPK) cascade that controls WUSCHEL (a master regulator of stem-cell fate in the shoot meristem) expression [34]. However, our knowledge about the potential link between ROPs and MAPKs is very limited (see Section 3.3). Whether CLAVATA1 interacts directly with the ROP G-protein in this complex or through another protein was not ascertained. The known components of the 450 $\mathrm{kD}$ complex - the disulphide-linked CLAVATA1 multimer, the kinase-associated protein 
phosphatase, and $\mathrm{ROP}$ - account for only $275 \mathrm{kD}$; therefore, there is room for several other unidentified components. RopGEFs were not yet known of at the time of the isolation of these protein complexes, and the direct regulation of ROPs via CLAVATA1-mediated phosphorylation was hypothesized [13]. However, based on the accumulating experimental evidence summarized below, it can be supposed that CLAVATA1 activates the ROP Gprotein indirectly, through a RopGEF.

During the 16 years that have elapsed since the publication of the CLAVATA1-ROP interaction [13], neither the association of a specific RopGEF with the CLAVATA1 complex nor the role of the ROP pathway in shoot meristem signalling have been clarified [34]. Based on microarray data, ropgef 2 has a strong expression in the shoot apex [35]. Therefore, it is a good candidate to associate with CLAVATA1-related receptor complexes, which could be verified using specific antibodies. The identification of rop or ropgef mutants with clavatal mutant-like phenotypes (enlarged shoot and flower meristems, additional lateral organs, and/or disrupted organ placements) could provide genetic evidence for the role of the potential CLAVATA1-RopGEF-ROP complex in shoot meristem maintenance. Null mutations of these genes might cause embryo lethality, similarly to the ropgef 7 mutation that affects the root meristem development negatively and results in non-viable embryos [25]. The silencing of rop and/or ropgef genes would be an alternative approach that could result in viable transgenic plants with decreased RNA levels, allowing the investigation of postembryonic phenotypes [25].

\subsection{POLLEN RECEPTOR KINASES}

The functional link between receptor kinases and ROP GTPases was first demonstrated in tomato plants during pollen tube growth. The tomato LeROP was present in a high molecular weight (approx. $400 \mathrm{kD}$ ) pollen protein complex, which also included the POLLEN RECEPTOR KINASEs (PRKs) [36]. These kinases are localized in the plasma membrane of 
pollen tubes, and they regulate pollen germination and tube growth [37]. The cytoplasmic domains of tomato PRKs - LePRK1 and LePRK2 - were used as bait in yeast two-hybrid screening, to help identify downstream targets from a tomato pollen cDNA library [16]. A pollen-specific protein with an unknown function, named "KINASE PARTNER PROTEIN", was identified as interacting with both kinase domains. The tomato KINASE PARTNER PROTEIN is equipped with the ROP nucleotide exchanger or PRONE domain, and belongs to the family of RopGEF proteins [38, 39]. Interaction between Arabidopsis PRKs and several RopGEFs was also demonstrated $[14,15]$. Considering the fact that RopGEFs are upstream positive regulators of ROPs (Fig. 1), the existence of PRK-RopGEF-ROP signal transduction pathways was hypothesized [38]. This hypothesis is now widely accepted, due to the accumulating experimental data discussed below (see also [9]).

Obviously, the potential for the phosphorylation of RopGEFs by PRKs was also investigated. The tomato KINASE PARTNER PROTEIN contains more than 25 predicted phosphorylation sites, and the two-dimensional electrophoresis of control and phosphatasetreated KINASE PARTNER PROTEIN indicated that at least one phosphorylated form of this protein exists in pollen [16]. Pollen-expressed RopGEFs of Arabidopsis have variable Cterminal sequences, which were implicated in the auto-inhibition of the biochemical activity of proteins dependent on their phosphorylation status $[14,15,39]$. The expression of truncated AtRopGEF12 missing the whole C-terminal part, but not the full-length version, disturbed pollen tube growth in a way reminiscent of ectopic RopGEF activity [14]. The Cterminal S510D phosphomimetic mutant of AtRopGEF12 resulted in similar phenotypes to those of the C-terminally truncated protein, when expressed in pollen tubes [14]. Furthermore, the S460A or S480A mutations, preventing the phosphorylation of the RopGEF1 protein at these C-terminal residues, abolished the in vivo functions of the protein: the mutant proteins failed to induce depolarization of pollen tube growth or even inhibit tube elongation [15]. 
Despite the fact that in vivo data about the phosphorylation of AtRopGEF12 - as well as AtRopGEF1 - are still missing, the phenotypes of the mutants mimicking or altering the potential phosphorylation sites of these proteins provide good support to the hypothesis that the function of pollen-expressed RopGEFs is controlled by protein kinases. Since PRKs interact with both AtRopGEF12 and AtRopGEF1 in vitro and in planta, they are good candidates for being responsible for this control. Several in planta functional studies support this hypothesis. The co-expression of AtRopGEF12 and the Arabidopsis PRK2a in pollen tubes resulted in isotropic growth (growth in all directions) instead of polar growth, reminiscent of the consequences of constitutively active ROP overexpression [14]. The same phenotype could not be observed, however, if the catalytically inactive mutant form of the kinase was co-expressed with the GEF protein. Therefore, this experiment supports the view that PRK2a-mediated GEF activation requires a phosphorylation step.

Further experiments have strengthened this interpretation: the expression of a dominant negative PRK inhibited the germination of Arabidopsis pollen, which could be rescued by overexpressing a constitutively active form of AtRopGEF1 [15]. Furthermore, AtROP1 activity at the pollen tip plasma membrane was positively regulated by the overexpression of the wild type, and negatively by that of the dominant negative mutant PRK [15]. These data are consistent with the existence of a PRK-RopGEF-ROP signalling module, regulating polar pollen tube growth in response to extracellular stimuli. However, the details of the functional PRK-RopGEF interaction need to be revealed by further experimentation.

Future experiments should include the identification of potential phosphorylation sites (e.g., via in vitro kinase assays, coupled with phosphopeptide identification) as well as biochemical and in planta studies revealing the functional consequences of RopGEF phosphorylation at the given residue(s). Furthermore, considering the large number of potential phosphorylation sites of RopGEFs, the involvement of kinases other than PRKs can 
be hypothesized in the control of RopGEF activity (see, e.g., Section 2.8). Phospho-specific RopGEF antibodies could help to highlight the spatial and temporal aspects of the kinasemediated regulation of RopGEFs in vivo. The first steps made in this direction are discussed below.

There is in vitro experimental evidence for PRK-mediated phosphorylation of AtRopGEF1, but the phosphorylation site is as yet undetermined [15]. A similar approach with AtRopGEF12 was unsuccessful [14], raising the possibility that the various RopGEFs are regulated in a number of different ways that may even include various kinases (see Section 2.8 for more details). This view is supported by the fact that the potential C-terminal phosphorylation sites of AtRopGEF1 and AtRopGEF12 are evolutionarily not conserved ([15], see also Fig. 2). These RopGEF proteins belong to different groups (Fig. 2) and differ from each other in several aspects. While the full-length AtRopGEF1 protein is not active in vitro, due to auto-inhibition by its own C-terminal region [15], this is not the case with the tomato KINASE PARTNER PROTEIN and AtRopGEF9 (which are both closely related to AtRopGEF12) [40]. The C-terminal regions of these two proteins exert auto-inhibitory activity only in vivo, not in vitro [40]. The C-terminal regions of AtRopGEF12-related proteins might be subjected to post-translational modification(s) in planta, for the efficient auto-inhibition of enzymatic activity. This requires the action of as-yet-unknown cellular factors. If these factors are also protein kinases, then the activity of AtRopGEF12-type proteins might be regulated positively (at the S510 residue; see earlier in this section), as well as negatively (at an as-yet-unknown site), by C-terminal phosphorylation.

The N-terminal regions of AtRopGEF1 and AtRopGEF12 might also serve different functions: the N-terminus of AtRopGEF1 contributes to the auto-inhibition of GEF activity, while that of AtRopGEF12 contributes to its enhancement $[14,15]$. The green fluorescent protein conjugated to RopGEF1 was localized at the pollen tube apex, while the other pollen- 
expressed RopGEFs were located in a more restricted area around the apical dome [39]. It is reasonable to suppose that the numerous pollen-specific RopGEFs govern specific but overlapping pathways. This is supported by the fact that only the ropgef $1,9,12,14$ quadruple mutant has defective pollen tube growth [15]. How the various RopGEFs contribute to the regulation of the spatio-temporal events governed by the various PRK receptor complexes is an interesting question still waiting to be answered (for more details, see [23]). Given the possession of specific antibodies, the in vivo interaction of given PRKs and RopGEFs could be analysed by "in situ" proximity ligation assays [41] at various phases of pollen tube growth, and in response to the various ligands bound to the receptors [23].

ROPs are locally activated at the pollen tube apex, where they regulate exocytosis [42]. In addition to the membrane and wall materials required for tip growth, exocytic vesicles also deliver upstream ROP activators, PRKs and RopGEFs to the apical plasma membrane [37, 42]. This way, ROP-focused exocytosis participates in a positive feedback loop, establishing and regenerating the active ROP protein pool at the pollen tube tip, required for the oscillatory growth of pollen tubes [42].

In addition to the indirect link between receptor-like kinases and ROPs through RopGEFs, the direct interaction of GTP-bound active tomato LeROP with a tomato PRK has been demonstrated [40]. Based on this ROP-conformation-specific interaction, which is a characteristic of ROP-effector kinases (e.g., [43-46]), the possibility was raised that the activity of PRKs might depend on an interaction with active GTP-bound ROPs [40]. Based on the strong interaction between AtRopGEF1 and AtROP1-GTP [15], an alternative hypothesis has also been developed. According to this theory, the active ROP G-protein might enhance the interaction between the PRK and the RopGEF, ensuring a positive feedback regulation [15]. In vitro kinase assays, using the kinase domain of the receptor kinases in the presence and absence of ROP-GTP, could help to decide which hypothesis is correct. 
The large number of pollen-expressed receptor-like kinases, RopGEFs and ROPs is in agreement with the fact that the pollen tube is capable of receiving and responding to various extracellular signalling cues [47]. Ligand-dependent, PRK-mediated phosphorylation and/or localization of specific RopGEFs may be the key step(s) in the spatial and temporal coordination of ROP-centred signalling pathways.

\subsection{FERONIA-type protein kinases}

FERONIA was identified as a RopGEF-interacting receptor kinase in an attempt to identify upstream regulators of ROP GTPase signalling in Arabidopsis [18]. A feronia mutant was found to have severe root hair growth defects among other phenotypes [18]. ROP activity is also required for polar root hair growth [48]. Therefore, it was hypothesized that FERONIA may activate ROP GTPase(s) through RopGEFs during this process. Several pieces of experimental evidence support this hypothesis: i) the feronia phenotype correlated with reduced levels of active ROPs; ii) the upregulation of ROP signalling counteracted the effects of feronia mutation; and iii) haemagglutinin epitope-tagged FERONIA and maltose-binding protein-tagged ROP2 (preferably the GDP-bound inactive form) interacted with each other in plant cells, as indicated by the co-immunoprecipitation of both proteins using either of the two antibodies which recognise the epitope tags [18]. FERONIA -dependent ROP activity could also be correlated with NADPH-oxidase-catalysed accumulation in roots and root hairs [18]. The role of ROPs in the spatiotemporal regulation of NADPH-oxidase-mediated reactive oxygen species (ROS) production has been shown previously [48]. Reactive oxygen species regulate hyperpolarization-activated $\mathrm{Ca}^{2+}$ channels [49] and thus contribute to the maintenance of a tip-focused $\left[\mathrm{Ca}^{2+}\right]$ gradient, which is fundamental for apical growth [50]. Therefore a full ROP-dependent signalling pathway, from a receptor (FERONIA) to an effector (NADPH oxidase), can be envisioned. 
FERONIA is a receptor of the non-glycosylated RAPID ALKALINIZATION FACTOR (RALF) 1 peptide [51]. The RALF1 peptide, similarly to FERONIA, is implicated in the regulation of plant growth. RALF1 binding results in FERONIA activation, and subsequently, in the phosphorylation-mediated inhibition of the plasma membrane $\mathrm{H}^{+}$-ADENOSINE TRIPHOSPHATASE 2, involved in proton transport [51]. Inhibition of proton transport results in the alkalinization of the apoplast and prevents cell elongation. The tip growth of root hairs is characterized by the oscillations of the apolastic $\mathrm{pH}$ right at the apex of the elongating hairs, which are likely to regulate cell wall extensibility and thereby cell expansion [52]. In tobacco, the silencing of a RALF-coding gene slowed apoplastic $\mathrm{pH}$ oscillations and increased the $\mathrm{pH}$ at the tips of root hairs, inhibiting their elongation [53]. Whether RALF1 or similar peptides are involved in the FERONIA-mediated activation of ROP G-protein signalling at the root hair tip is unknown at present. One can speculate that RALF1 regulates tip growth, controlling apoplastic $\mathrm{pH}$ oscillations in parallel with the ROP-mediated establishment of $\left[\mathrm{Ca}^{2+}\right]$ gradient at the root hair apex. In tobacco, the prevented accumulation of a RALF peptide decreased the accumulation of reactive oxygen species in the root hair initiation zone, indicating the possible involvement of RALF-type peptides in reactive oxygen species generation [53]. Whether this regulation involves ROP G-protein-mediated NADPHoxidase activation could be decided by investigating this response in mutants defective in RopGEF function. However, we cannot exclude the idea that FERONIA might be activated in a coordinated manner by several different ligands during the regulation of root hair initiation and elongation. In addition to root hair growth, the FERONIA receptor is involved in a variety of developmental processes and was hypothesized to have several different ligands associated with its various functions [54].

Which of the several AtROPs and AtRopGEFs could play specific roles in FERONIAmediated signalling during root hair growth in Arabidopsis? Loss- and gain-of-function 
analyses have indicated that AtRopGEF4 and AtRopGEF10 are the RopGEFs involved in the root hair growth pathway [17]. While AtRopGEF4 is important for root hair elongation, AtRopGEF10 mainly contributes to hair initiation [17]. Consistently, the gef4gef10 double mutation led to roots with shorter and less dense root hairs. This correlated with strongly decreased active ROP levels, as well as lower reactive oxygen species levels, in the mutant roots. AtROP2 and AtROP6, which promote root hair growth, interacted with both AtRopGEF4 and AtRopGEF10; however, AtROP7, which inhibits root hair growth, did not interact with either of these [17]. The significant (yet not total) absence of both GEF4 and GEF10 prevented the FERONIA overexpression-mediated production of reactive oxygen species. Interestingly, while the feronia-4 mutation prevented the increased initiation and growth of root hairs in response to exogenous auxin or reduced phosphate levels, the gef4 and gef10 single and double mutants reacted as the wild type [17]. Therefore, it was hypothesized that, although FERONIA is required for both the developmental and the environmental regulation of root hair growth, these latter pathways are RopGEF4- and RopGEF10independent [17]. This view has been supported by two observations. On the one hand, the FERONIA overexpression-mediated increase in the level of reactive oxygen species was not completely abolished in the gef4gef10 double mutant background; on the other hand, the gef4gef10 mutant had a milder phenotype than the feronia-4 mutant [17]. These results imply that AtRopGEF(s) other than AtRopGEF4 or AtRopGEF10 are involved in the FERONIAdependent auxin response pathway. Therefore, it is plausible to suppose that various developmental and environmental signals converge in different FERONIA-containing receptor complexes that have distinct RopGEFs and/or ROPs.

This view has recently been strengthened by a study connecting FERONIA to abscisic acid signalling [21]. Based on protein-protein interaction and genetic studies, an Arabidopsis FERONIA signalling pathway_-including the AtRopGEFs GEF1, GEF4 and GEF10, and the 
ROP GTPase AtROP11 — was established [19-21, 55]. Mutations disrupting any member(s) of this pathway, including feronia, geflgef4gef10 and rop 11, resulted in hypersensitivity to abscisic acid, indicating the role of the FERONIA pathway in the suppression of abscisic acid signalling. Abscisic acid-induced ROS accumulation strongly increased in the auxin responsedefective [18] feronia-4 mutant [21]. Therefore, FERONIA pathways seem to be involved in both abscisic acid- and auxin-induced NADPH oxidase-mediated ROS production, providing the possibility for a crosstalk between these two frequently antagonistic plant hormones. The expression of fer itself is oppositely regulated by auxin and abscisic acid, as auxin upregulates while abscisic acid downregulates fer transcription [21]. These hormones both control the transcription of the Arabidopsis rop9 GTPase; however, AtROP9 negatively regulates the auxin and positively regulates the abscisic acid responses [56]. Whether AtROP9 signalling opposes the FERONIA-RopGEF1,4,10-ROP11 signalling pathway is a question yet to be answered [56]. Revealing the upstream regulation of AtROP9—-possibly including receptorlike kinases and RopGEFs - and the phenotype caused by the overexpression of the constitutively active AtROP9 GTPase, could provide important further insights into the auxinabscisic acid crosstalk.

The first identified role of FERONIA was in fertilization. Its loss-of-function resulted in pollen tube overgrowth within the embryo sac [57]. Pollen tubes are normally attracted by feronia mutant female gametophytes, but pollen tubes reaching the synergid do not rupture and therefore cannot release sperm, meaning that fertilization fails. The accumulation of ROS required for pollen tube rupture is dependent on FERONIA [22]. Inhibiting ROS production in the gametophyte has the same phenotypic effect as the feronia mutation on pollen tube growth. A high exogenous ROS level probably weakens the pollen tube wall, promoting tube rupture. It has been suggested that FERONIA regulates female gametophytic ROS production through a similar pathway that operates during root hair growth, and involves RopGEFs, 
ROPs and the plasma membrane NADPH oxidase [22, 58]. One possible way in which FERONIA could regulate downstream signalling is through the direct phosphorylation of RopGEFs. However, even though the kinase domain of FERONIA is necessary for pollen tube reception, kinase activity is not [59]. Consequently, FERONIA is hypothesized to serve as a co-receptor, recruiting other factors: potentially other kinases mediating the extracellular signals towards cellular targets [59].

The ROS-induced pollen rupture is $\mathrm{Ca}^{2+}$-dependent. This may explain why the pollen tube disintegrates after entering the synergid [22]. The $\mathrm{Ca}^{2+}$ level in the synergid cytoplasm is rather high and increases with the pollen tube's arrival. This increase could be evoked by FERONIA via ROPs and/or ROS, since both regulate $\mathrm{Ca}^{2+}$ channels. The tip-located NADPH-dependent ROS production required for pollen tube growth also increased in response to exogenous $\mathrm{Ca}^{2+}[60]$. Based on these observations, it was hypothesized that the already ROS-challenged pollen tube responds to the high intracellular synergid $\mathrm{Ca}^{2+}$ level with further ROS production, leading to a ROS-induced $\mathrm{Ca}^{2+}$ channel opening and a sudden water influx that finally results in the pollen tube bursting [22].

This model is in agreement with the fact that pollen tubes survive the high ROS environment at the filiform apparatus, but burst instantaneously within the synergid, due to the presence of $\mathrm{Ca}^{2+}$. The pollen tube also has its own control on the process, via the FERONIA-related ANXUR receptor-like kinases. While FERONIA is widely expressed in the plant, except in the pollen, its closest homologues ANXUR1 and 2 are pollen-specific [27]. The complementary expression patterns of FERONIA and ANXUR1/2 fit well with their contrasting roles in regulating pollen tube rupture and integrity, respectively. The disruption of ANXUR1 and ANXUR2 in double mutants triggers precocious pollen tube ruptures in the pistil, and therefore the sperm cells never reach the ovule [26]. ANXUR1 and 2 are the regulators of pollen-expressed NADPH oxidases that generate superoxide radicals at the tip, 
which are converted into $\mathrm{H}_{2} \mathrm{O}_{2} \cdot \mathrm{H}_{2} \mathrm{O}_{2}$ accumulation affects the $\mathrm{Ca}^{2+}$ channels and thus contributes to the maintenance of the tip-focused $\mathrm{Ca}^{2+}$ gradient during pollen tube growth [26].

ANXUR1 and 2 positively regulate exocytosis and the secretion of cell wall material [26]. It is hypothesized that ANXUR1 and 2 maintain pollen tube cell wall integrity until the tube reaches the synergid, where their function is disengaged, promoting FERONIA-dependent and exogenous ROS- and $\mathrm{Ca}^{2+}$-mediated rupture. It has also been hypothesized that FERONIA (on the synergid) and ANXUR1/2 (on the pollen tube surface) may compete for the same ligand that coordinates activation/inactivation [58]. The anxurl anxur2 double mutant pollen tube loses polarity and bursts, in a similar way to the feronia loss-of-function mutant root hair $[18,26]$. Given the similarities between ANXUR1/2 and FERONIA in the maintenance of cell wall integrity and tip growth, via NADPH oxidase-dependent ROS production, similar signalling pathways for these receptor kinases can be envisaged [58]. However, the link between ANXUR1/2, and RopGEFs and ROPs, still awaits experimental confirmation.

If the tomato PRK2 expression is suppressed in pollen tubes, the $\mathrm{Ca}^{2+}$-induced apical ROS production is compromised [61], suggesting that the ANXUR1/2- and PRK-regulated pathways are interrelated [27].

FERONIA is a widely expressed, multifunctional protein involved in many aspects of plant development. In addition to root hair and pollen tube growth, FERONIA is involved in brassinosteroid signalling, hypocotyl elongation, and powdery mildew infection, among other functions (for a review, see [62]). Although details of these FERONIA-mediated signalling pathways have not yet been elucidated, one can suppose that the RopGEF-ROP-NADPH oxidase signalling module that controls $\mathrm{H}_{2} \mathrm{O}_{2}$ and $\mathrm{Ca}^{2+}$ levels is involved in most, if not all, 
FERONIA-dependent pathways. Arabidopsis FERONIA controls seed size by inhibiting integument cell elongation in a RopGEF-dependent way that further supports this view [23].

Mutations in the genes of several other FERONIA-type receptor kinases-e.g., THESEUS, HERKULES and ERULUS-also interfere with cell elongation, cell wall integrity, and ROS-related functions [54, 63]. These kinases may also serve as upstream regulators of ROP GTPase signalling. While the kinase domains of three members of the family (FERONIA, ANXUR1 and HERKULES1) were interchangeable in an in planta functional assay, their extracellular domains were not [59]. Therefore, these receptor kinases may transduce various external signals towards the same or similar targets. There are only unpublished data to support the interaction of FERONIA-related kinases other than FERONIA with RopGEF proteins (cited in [63]). Only extensive future research can reveal the spatio-temporal interaction patterns of the various FERONIA-type receptors, their ligands, RopGEFs, ROPs, and NADPH-oxidase proteins in the development and environmental responses of plants. The engineering of constitutively active RopGEF versions (e.g., by removing the C-terminal auto-inhibitory domain; see Section 2.2) could be used for the complementation of receptor mutants, to support their functional interaction in planta. Potential receptor kinase-RopGEF pairs could be hypothesized based on the similarities between mutant phenotypes, coupled with co-expression analysis and traditional proteinprotein interaction assays. However, these approaches might prove difficult, due to the similar structures and overlapping functions of the members of these protein families.

\subsection{The TRANSMEMBRANE KINASE receptor kinases}

ROP GTPases play a central role in leaf epidermal tissue patterning, spatially coordinating the expansion of neighbouring pavement cells resulting in a jigsaw-puzzle-like appearance of the epidermis [64]. Auxin also promotes the formation of interdigitated epidermal cell patterning in a spatio-temporally regulated manner [65]. It was hypothesized that auxin- 
activated ROPs regulate cytoskeletal organization and endocytosis, as well as the recycling of the auxin efflux carrier PIN-FORMED proteins [65]. Auxin could therefore serve as a selforganizing signal, causing the polarization of the PIN-FORMED proteins that are required for polar auxin transport. The established auxin gradients finally determine the characteristic planar polarity of the epidermal cells. Certain Arabidopsis ROPs, (ROP2/4 and ROP6) have been implicated in the process [65]. The interdigitation of pavement cells is compromised in rop2-RNAi rop4-1, as well as in auxin synthesis mutants. Auxin application could not rescue this defect of the rop2-RNAi rop4-1 mutant, indicating the role of ROP2/4 in auxin sensing [65]. There is not yet any consensus on how the auxin signal is transduced in order to activate ROPs. According to a recent hypothesis, the AUXIN-BINDING PROTEIN 1 (ABP1) represents the link. ABP1 is widely considered to serve as an auxin receptor, as it has been shown to reversibly and specifically bind auxin with high affinity and to trigger auxinassociated physiological responses (for a recent review, see [66]). Although the first identified T-DNA insertion mutant of ABP1 was embryo lethal, several techniques have been applied to increase or decrease the level of this protein in plants. These approaches have resulted in appropriately altered auxin responses, supporting the role of ABP1 as an auxin receptor [66].

This partly apoplastic auxin-binding protein (it has a larger pool in the endoplasmic reticulum) was suggested to transduce the auxin signal towards the cytoplasm, through an interaction with members of the TRANSMEMBRANE KINASE (TMK) receptor kinase family [28]. Arabidopsis plants producing the mutant ABP1 version that cannot bind auxin and those expressing tmk1,2,3,4 quadruple mutants have similar phenotypes: abnormal PINFORMED1 localization, embryo lethality, single cotyledons, and fused leaf-cups [28]. Moreover, auxin promotes a complex formation between ABP1 and TMK1, in an auxin concentration-dependent way [28]. Therefore, the hypothesis was established that the secreted ABP1 binds auxin and subsequently associates with the extracellular domain of TMK1, which 
transduces a signal towards the cytoplasm [28]. However, two different abpl null mutants

were described in a recent report, and these mutants had neither developmental abnormalities nor auxin resistance [67]. Therefore, the role of the ABP1 as an auxin receptor has to be reinvestigated, despite the extensive, previously accumulated experimental evidence supporting this function. For example, the various methods used to alter the expression or function of ABP1 could be re-evaluated in the null mutant background, in order to see whether they cause phenotypic changes in the absence of ABP1.

Nevertheless, auxin-mediated ROP activation was largely abolished in the transmembrane kinase mutants, suggesting that these receptors are part of the signal transduction chain, even if ABP1 is not [28]. It is reasonable to suppose that RopGEFs can serve as direct TMK targets, linking this cell surface receptor to ROP GTPase signalling. SPIKE1, the only plant protein belonging to the DHR2-Dock family of RhoGEFs, was implicated in auxin-regulated pavement cell morphogenesis [8], as well as in auxin perception, PIN-FORMED2 internalization, and ROP6 activation in roots [29]. Therefore, SPIKE1 could be a primary candidate for transferring the TMK signal towards ROP GTPases. The direct interaction of TMKs with SPIKE1 would require the presence of both proteins at the plasma membrane. Although this possibility cannot be ruled out completely, most of the SPIKE1 protein accumulates at specific endoplasmic reticulum domains, as well as on punctate internal organelle surfaces, where it co-localizes with ROPs [68]. Therefore, TMKs might increase SPIKE1 and ROP activities indirectly through the activation of an intracellular signalling cascade. How the endoplasmic reticulum-related activation of ROPs can coordinate cell growth at the cell cortex is currently unknown [68]. It was hypothesized that SPIKE1 might regulate the early secretory pathway and the partitioning of ROP-GTP into specialized membrane domains [68].

\subsection{The PANGLOSS1 maize receptor-like kinase}


That the Cdc42 Rho-type small GTPase regulates asymmetric cell division in yeast and animal cells has long been known, but a similar role for plant ROPs had not been elucidated until recently. Guard cell development is used as a plant model to study the regulation of cell division asymmetry. Extrinsic clues orient the cell division polarities occurring in an invariant sequence, which generate the stomatal complexes of maize leaves. These complexes consist of a pair of guard cells, flanked by a pair of subsidiary cells regulating the stomatal aperture. During the process, the guard mother cell signals to its neighbours, the subsidiary mother cells, to divide asymmetrically in an orientation that positions the smaller daughter cell (the subsidiary cell) adjacent to the guard mother cell. The subsidiary mother cell division is preceded by the asymmetric localization of cortical F-actin at the contact site between the guard and subsidiary mother cells respectively, followed by the migration of the subsidiary mother cell nucleus towards that site. A leucine-rich repeat receptor-like kinase, PANGLOSS1 was identified as a potential subsidiary mother cell receptor for guard mother cell-derived polarizing cues [69]. The ROPs (maize ROP2 and 9) function together with PANGLOSS1 in this process [31]. ROPs, such as PANGLOSS1, accumulate asymmetrically in subsidiary mother cells and promote the localized accumulation of F-actin at the guard mother cell-contact site. The asymmetric ROP localization depends on PANGLOSS1, indicating that ROPs function downstream of this receptor. Although a direct physical interaction between ROPs and PANGLOSS1 could not be demonstrated, they are both present in the same protein complex, co-immunoprecipitated from maize cells. It is reasonable to speculate that RopGEFs mediate the interaction between PANGLOSS1 and ROPs, similarly to other ROP-containing receptor-like kinase complexes in Arabidopsis (see Sections 2.22.4).

Sequence analysis and in vitro experiments indicate that PANGLOSS 1 is inactive as a kinase [69]. Therefore, PANGLOSS1 may serve as a scaffolding protein, correctly 
positioning polarity-related protein complexes containing the ROPs, among other components. While the ectopic overexpression of ROP2 causes aberrantly oriented subsidiary mother cell divisions, the partial loss of ROP2/ROP9 function causes a weak subsidiary mother cell division polarity phenotype, strongly enhanced by the pangloss 1 mutation. Consequently, ROPs may also have PANGLOSS1-independent functions in subsidiary mother cell polarization.

\subsection{The LYSIN MOTIF DOMAIN RECEPTOR-LIKE KINASES}

OsRAC1, a rice ROP GTPase, plays an important role in rice innate immunity [70]. The constitutively active form of OsRAC1 is contained within a large protein complex, implicated in the recognition of pathogen-associated molecular patterns (for reviews, see $[71,72]$ ). This "defensome" complex [71] contains two cell surface pattern recognition receptors: the lysin motif-domain CHITIN OLIGOSACCHARIDE ELICITOR-BINDING PROTEIN and the lysin motif-domain CHITIN ELICITOR RECEPTOR KINASE 1 [32]. CHITIN OLIGOSACCHARIDE ELICITOR-BINDING PROTEIN is a receptor-like protein with no intracellular domain, while CHITIN ELICITOR RECEPTOR KINASE 1 is a functional kinase that does not bind chitin [32]. These two receptor proteins form a complex that is capable of transducing the chitin signal towards downstream components, including OsRAC1. In response to chitin, the activity of OsRAC1 is quickly induced. The complex also contains OsRacGEF1, which interacts with the OsCHITIN ELICITOR RECEPTOR KINASE 1. The kinase phosphorylates the C-terminal part of OsRacGEF1 at the S549 residue, which is required for efficient OsRAC1 activation. The GTP-bound OsRAC1 promotes ROS production, lignin biosynthesis and protein kinase signalling through its downstream targets: the NADPH oxidase, CINNAMOYL-COA REDUCTASE 1, and MAPK6, respectively.

In addition to pathogen defence, lysine motif-containing receptor kinases are involved in symbiotic plant-pathogen interactions, in which ROP GTPases also play a role [73]. The 
expression of the Lotus japonicus ljrop6 gene is elevated after the inoculation of roots with Mesorhizobium loti, the symbiotic bacterial partner of Lotus japonicus [73]. Moreover, the loss of ROP6 function due to RNA-interference inhibits the growth of the infection thread, resulting in fewer root nodules [73]. The kinase domain of the lysine motif domain-containing NOD FACTOR RECEPTOR 5 receptor kinase binds the LjROP6 GTPase [33]. NOD FACTOR RECEPTOR 5 is likely to be a non-functional enzyme, as it has no activation loop in the kinase domain, but interacts with a functional receptor kinase: NOD FACTOR RECEPTOR 1. The NOD FACTOR RECEPTOR s might be involved in LjROP6-mediated signalling through the activation of a yet unknown RopGEF, which might be phosphorylated by NOD FACTOR RECEPTOR 1.

\subsection{Further RopGEF-ROP signalling modules potentially linked to receptor kinases}

There are several further reports on RopGEF-ROP signalling interactions with as-yetunknown upstream receptors (Table 2). Local activation of the Arabidopsis ROP11 GTPase is dependent on RopGEF4 during the formation of secondary cell walls in the development of xylem cells [74]. It was hypothesized that the regulation of secondary cell wall patterning might rely on receptor-like kinases acting upstream of the RopGEF-ROP module [30]. The Arabidopsis RopGEF7 interacting with ROP3 is required for polar auxin transport and meristem maintenance in the root [25]. Considering their overlapping expression patterns and direct interaction in a yeast two-hybrid assay, the FERONIA receptor kinase has been proposed as a potential upstream regulator of RopGEF7 [18, 24]. The ROP nucleotide exchange factor SPIKE1 was also implicated in the establishment of polar auxin transport in the root, together with the ROP6 GTPase [29]. The auxin signal-perceiving TRANSMEMBRANE KINASEs, ROP GTPases, and the polar auxin transport were demonstrated as acting together in the patterning of the leaf epidermis ([28]; see Section 2.4), and the existence of a similar pathway can be predicted to function in auxin-transporting root 
cells. The co-expression pattern of receptor kinase and RopGEF genes (e.g., [35]), along with pairwise protein-protein interaction assays, can help us to elucidate which receptor-RopGEF pair plays a role in a given process. However, monitoring receptor signalling in RopGEF mutant backgrounds can only give a strong indication that there is a functional link between the proteins. Considering the redundant function of several RopGEFs in a given process (see examples in Table 2), multiple RopGEFs would have to be mutated in many cases, in order to see a clear difference in a signalling response.

\subsection{Non-receptor protein kinases upstream of ROP GTPase signalling}

The above-described examples indicate that the receptor-like kinase-RopGEF-ROP signalling module is widely utilized during plant development and defence. However, the modulation of ROP-signalling can also be achieved through the phosphorylation of either the ROPs themselves or their negative regulators, including the ROP GTPASE ACCELERATING PROTEINs (RopGAPs) and the ROP GUANINE NUCLEOTIDE DISSOCIATION INHIBITORs (RopGDIs) (Fig. 3). Although receptor kinases might also phosphorylate these targets, the available data implicate non-receptor kinases-such as MITOGEN-ACTIVATED PROTEIN KINASEs (MAPKs) or CALCIUM-DEPENDENT PROTEIN KINASEs (CPKs) —in these processes, as discussed below (see also Fig. 3).

There are indications that ROPs, similarly to animal Rho-type GTPases, may also be regulated through direct phosphorylation. In animal cells, RhoA, Rac1 and Cdc42 all undergo phosphorylation by specific protein kinases at various residues [75]. The phosphorylation of these proteins can increase stability, alter cellular localization, inhibit nucleotide binding or change the interaction with upstream regulators or downstream effectors [75]. One of the phosphorylation sites (S71YP in human Rac1) is well conserved in all Rho proteins, including plant ROPs (S74YR in Type I ROPs; [76, 77]). The residues in the close vicinity of this putative phosphorylation motif are required for the interaction of ROPs with RopGEFs [77]. 
The phosphomimetic (S74E) mutation of the evolutionarily conserved serine residue in Medicago ROP6, as well as in Arabidopsis ROP4, altered the binding interface of the two proteins and prevented the ROP-RopGEF interaction in vitro as well as in vivo [76]. Consequently, the phosphorylation of S74 may serve as a negative regulatory event, preventing ROP activation by RopGEFs. The biological significance of this potential regulatory step is as yet unknown and the kinase that may carry out the phosphorylation is not yet identified. In certain cases, the direct binding of ROPs to the kinase domain of receptor kinases could be observed [40], but no direct phosphorylation of ROPs by these kinases has been reported so far. The human Rac1 protein is phosphorylated on the corresponding residue by the AKT kinase and this phosphorylation is inhibitory [78]. However, this region is not involved in the binding of Rac1 to its regulatory GEFs [77]. The AKT-mediated inhibition of Rac1 is exerted through the decreased GTP-binding ability of the phosphorylated G-protein [78]. AKT belongs to the large AGC kinase family that is also present in plants [79]. AGC kinases are implicated in many plant development and defence pathways also involving ROP GTPases, such as root hair and pollen tube growth, auxin transport, etc. [79]. Considering their functional relationship and the conservation of the S71 residue in plant ROPs, as well as in the human RAC1 protein, AGC-type kinases are candidate ROP kinases in plants (Fig. 3). This potential regulatory link still needs experimental validation. The in vitro phosphorylation of wild-type Type I plant ROPs by AGC kinases, but not the S74A mutant, could be an indication of a potential in planta signalling interaction. The effect of pollen-expressed AGC kinases on ROP activation could be tested in a pollen tube growth assay. ROP overexpression in pollen tubes results in the widening of the pollen tube tip, due to the ectopic ROP activation by pollen-expressed RopGEFs. Co-expression of AGC kinase with the ROP GTPase should prevent this activation as well as the tip-widening phenotype, in a similar manner to the S74E phosphomimetic mutation (e.g., [76]). 
Two of the 11 Arabidopsis ROPs (ROP4 and ROP7) contain MITOGEN-ACTIVATED PROTEIN KINASE (MAPK) docking motifs [80], suggesting their interaction with MAPKs. Indeed, ROPs and MAPKs might be present in the same protein complex [81], but as ROPs lack the MAPK phosphorylation consensus motif, they are unlikely to serve as direct MAPK targets [80]. However, all Arabidopsis RopGEFs possess both the docking and the phosphorylation motifs, and therefore were hypothesized to be phosphorylated by MAPKs [80]. The potential MAPK phosphorylation sites on the C-terminal regulatory regions of Arabidopsis and rice RopGEFs are shown in Fig. 2. While a few of these sites are present on all or most of RopGEFs, indicating a general regulatory function, others are conserved only in the homologous Arabidopsis and rice proteins, and there are yet others that seem to be unique to certain members, suggesting a regulatory role in specific processes. One of the evolutionarily conserved sites, the S510 of AtRopGEF12, has been hypothesized as being phosphorylated by the AtPRK2, but so far experimental evidence has not been provided (see Section 2.2 and Fig. 2). Therefore, we cannot exclude the idea that this MAPK consensus sequence is indeed targeted by a MAPK. It is also possible that this GEF-activating phosphorylation can be exerted by both types of kinases and therefore may serve as an integrator of various signalling pathways. The theory that MAPKs can modulate ROP GTPase signalling through RopGEF phosphorylation needs experimental validation in the near future. In vitro kinase assays could strengthen the hypothesis that RopGEFs are indeed potential MAPK substrates. In particular, these tests should consider those MAPKs known to be plasma membrane-localized [80], since in many cases, RopGEF signalling is initiated by plasma membrane-bound receptor kinases (see Section 2.1-2-7).

In addition to positive regulators like RopGEFs, negative factors - such as ROP GTPASE ACCELERATING PROTEINs and ROP GUANINE NUCLEOTIDE DISSOCIATION INHIBITORs - also control ROPs (Fig. 1). Only one of the RopGAPs bears MAPK 
phosphorylation signatures [80]. This RopGAP (gene code At5g61530), which has a strong pollen-specific expression, is the sole member of an Arabidopsis RopGAP family with no other domains but the catalytic domain [3]. After the in vitro verification of phosphorylation, the expression of phosphomimetic or non-phosphorylatable mutant versions of the protein in pollen tubes could give an indication of the existence and biological significance of this potential regulatory step. This could be completed using biochemical tests characterizing the enzymatic activity of the phosphorylated (or phosphomimetic mutant) protein.

One Arabidopsis ROP GTPASE ACCELERATING PROTEIN, designated as "ROP ENHANCER 1" (REN1), prevents the lateral propagation of apical ROP1 activity [82]. The loss of REN1 function in the ren1-1 mutant resulted in severely depolarized pollen tubes, similar to the results of the constitutively active ROP1 mutant overexpression. However, pollen tube growth in a weak renl allele (ren1-3) was normal at medium $\mathrm{Ca}^{2+}$ levels $(2-5$ $\mathrm{mM})$, but became depolarized under low extracellular $\mathrm{Ca}^{2+}$ conditions $(\leq 0.5 \mathrm{mM})$, suggesting that REN1 and $\mathrm{Ca}^{2+}$ may act together [82]. ROP1 activation promotes the tip-directed targeting of REN1 to the apical region and directly regulates the formation of tip-localized $\mathrm{Ca}^{2+}$ gradients. Therefore, ROP1, REN1 and $\mathrm{Ca}^{2+}$ were hypothesized to work together in a REN1-based negative feedback loop [82, 83].

CALCIUM-DEPENDENT PROTEIN KINASEs (CPKs) expressed in pollen have been proposed to mediate the $\mathrm{Ca}^{2+}$ response to REN1 activity [83]. Pollen-expressed CPKs (especially CPK17 and 34) are required for pollen tube growth and guidance in Arabidopsis [84]. Their potential substrates, however, are as yet unknown. In vitro assays could be used to determine the potential CPK phosphorylation sites of REN1. Mutating the phosphorylated residue to alanine or a charged (phosphomimetic) amino acid (e.g., glutamic or aspartic acid) could be used to test the potential role of REN1 phosphorylation on its cellular localization and tip growth-related function. 
The phosphorylation of Arabidopsis ROP GUANINE NUCLEOTIDE DISSOCIATION INHIBITOR1 by CPK3 affects ROP function negatively [85]. AtROP GUANINE NUCLEOTIDE DISSOCIATION INHIBITOR1 plays a role in leaf epidermal pavement cell formation, by interacting with AtROP2 and AtROP6 [85]. CPK3 phosphorylates three conserved serine residues in AtROP GUANINE NUCLEOTIDE DISSOCIATION INHIBITOR1 [85]. Overexpression of the triple-phosphomimetic-but not the nonphosphorylatable_-mutant protein could rescue a gdi1-1 mutant Arabidopsis seedling with an abnormal growth phenotype, indicating that phosphorylation is required for the proper GDI function.

Tobacco NtROP GUANINE NUCLEOTIDE DISSOCIATION INHIBITOR2 plays a role in the recycling of the NtRAC5 ROP GTPase, from the flanks of the tip to the apex of the pollen tubes, and in this way contributes to the polarization of ROP signalling. This protein was identified among the phosphoproteins present in in vitro activated tobacco pollen [86], suggesting that it might be regulated by a protein kinase. Considering the fine-tuned interrelation between calcium- and ROP-mediated signalling in pollen tubes $[83,87,88]$, one may envisage that pollen tube-expressed CPKs might be involved in GDI regulation. While the CPK-mediated phosphorylation regulates positively, its interaction with PHOSPHATIDYLINOSITOL 4-PHOSPHATE 5-KINASEs (PIP5Ks) affects the function of ROP GUANINE NUCLEOTIDE DISSOCIATION INHIBITORs s negatively during tip growth (see Section 3.4 for details).

\section{Protein kinases regulated by ROP GTPase signalling}

Given the involvement of Rho-type GTPases in a wide variety of important cellular processes, it is not surprising that they have a large number of cellular targets as well as effector proteins. Plant ROPs evolved independently of animal Rho-type GTPases, and 
formed a structurally unique group within the eukaryotic Rho family [2-4]. They have several downstream targets that are plant-specific (see examples in Table 1) and play roles in plantspecific processes, such as cell wall synthesis, auxin signalling and plant-pathogen interactions [2-4]. While there are over 100 metazoan Rho GTPase effector proteins, which include approximately 30 kinases - many of them carrying a Cdc42-Rac interactive binding (CRIB) motif $[1,11]$ —our present knowledge on ROP-interacting protein kinases in plants is rather scarce. Based on the available genome sequence data, we can state that plants do not possess protein kinases with the CRIB motif. However, plants do possess a family of CRIB motif-containing small adaptor proteins, named accordingly as "ROP-INTERACTIVE CRIB MOTIF-CONTAINING PROTEINs" [89]. It can be supposed that these ROPINTERACTIVE CRIB MOTIF-CONTAINING PROTEINs (RICs) link ROPs to downstream kinases in plants. Despite the accumulating evidence that ROPs use RICs as their direct effectors in a variety of pathways (e.g., [4]), the molecular events downstream of RICs are still shrouded in obscurity. The only report of a potential link between RICs and downstream kinases comes from lilies, in which staurosporin — a serine/threonine kinase inhibitor — blocks the inhibitory effect of the LLP12-2 RIC on pollen tube growth [90]. In addition to RICs, there are other types of ROP effector scaffold proteins (for reviews, see [2-4]); however, as yet there is no indication that they are linked to kinase signalling.

Plants do not have Ras GTPases, which regulate MAPK-cascades in animal cells. Therefore, ROPs are the only signalling-type small GTPase proteins in plants, and it is reasonable to suggest that they signal towards downstream kinases. The identification of kinases that directly interact with ROPs strongly supports this possibility [43-46]. The present view on the kinases potentially implicated in signalling downstream of ROPs is summarized below (see also Fig. 4).

\subsection{The ROP-activated RECEPTOR-LIKE CYTOPLASMIC KINASES}


Several ROP-interacting kinases have been identified in yeast two-hybrid protein-protein interaction screens, using constitutively active ROP/RAC GTPases as baits. These kinases include RECEPTOR-LIKE CYTOPLASMIC KINASEs (RLCKs) from Medicago truncatula [44] and Arabidopsis [43, 44, 46], as well as barley [45]. RLCKs, based on the sequence of their kinase domain, belong to the large receptor-like kinase family. Still, the majority of them do not possess either extracellular receptors or transmembrane domains [91]. RLCKs are classified into several distinct families, including 147 proteins in Arabidopsis and 379 in rice. Despite their large number, there is only very limited information available on the biological function of these kinases. Several of them have been implicated in associating with and transducing signals from receptor-like kinases during a variety of cellular processes, including hormonal and developmental regulation, as well as defence responses [91].

The RLCK VI family from Arabidopsis consists of 14 members, forming two subfamilies with seven members each [92]. The members of the RLCK VI_A subfamily [43, 44, 46], as well as their homologues from Medicago truncatula [44] and barley [45], bind to GTP-bound active - but not to the GDP-bound inactive-ROP GTPases in vitro, in yeast, and in planta. This GTPase conformation-specific interaction is characteristic of effector proteins. The in vitro protein phosphorylation activity of these kinases is also dependent on the presence of GTP-bound or constitutively active ROP/Rac GTPases [44-46], and therefore they can be considered to be ROP-effector kinases in a biochemical sense. However, the biological function of the RLCK VI_A family kinases is largely unknown. The barley ROP BINDING KINASE 1 (HvRBK1) plays a role during the infection process of powdery mildew (see below for details), a process known to be dependent on barley ROP/Rac GTPases [45]. The infection of barley leaf cells by powdery mildew is enhanced by the transient overexpression of constitutively active HvRACB, HvRAC3 or HvRAC1 ROP GTPases [93]. The transient knockdown of RBK1 in barley leaves similarly supported the penetration of the parasitic 
fungus, suggesting some function of the ROP-activated kinase in basal disease resistance [45]. The transient silencing of HvRBK1 weakened the stability of cortical microtubules, which is consistent with HvRBK1 having a function in building penetration barriers against the fungus [45]. Green fluorescent protein-tagged HvRBK1 appeared in the cytoplasm and nucleoplasm, but constitutively active HvRACB or HvRAC1 was able to recruit the tagged HvRBK1 protein to the cell periphery [45]. Therefore, barley HvRACs and HvRBK1 may function together in cortical microtubule dynamics, during the interaction with invading fungal pathogens. The silencing of the closest Arabidopsis homologue of HvRBK1, AtRLCK VI_A3, supported fungal susceptibility in a similar way to its barley counterpart [46]. Moreover, Arabidopsis RLCK VI_A4 (also called AtRBK1) and RLCK VI_A6 (AtRBK2) proteins are implicated in pathogen defence, based on their augmented gene expression in response to fungal infection [43].

Several of the Arabidopsis RLCK VI_A kinases have a strong pollen-specific expression [92], indicating their role in the ROP-mediated regulation of pollen tube growth. Furthermore, they are upregulated during the differentiation of tracheary elements [43], as well as being strongly dependent on ROP-regulated microtubule organization (see, e.g., [74]). Recently, the expression of the RLCK VI_A7 kinase gene (At5g18910) has been shown to be positively associated with the common gene expression profile of root hairs and pollen tubes, where ROP GTPases play a central role in regulating tip growth [94]. These observations indicate interrelations between ROP- and RLCK VI_A kinase-mediated pathways, but the dependence of the in planta function of RLCK VI_A kinases on their interaction with active ROP GTPases still awaits experimental confirmation. The production of transgenic plants, overexpressing constitutively active ROP GTPases in a RLCK VI_A loss-of-function background (e.g., due to the mutation or silencing of RLCK VI_A genes), could be used to obtain this confirmation. The overexpression of constitutively active ROP GTPases results in 
a wide range of phenotypes, including abnormal growth, morphogenesis and hormonal responses (e.g., [95]). Mild or non-developing phenotypes, in the absence of RLCK VI_A function, would indicate the role of the given kinase downstream of the given ROP GTPase in that process. The overlapping, redundant functions of the various ROPs, as well as the various RLCK VI_A kinases, make this approach challenging. Co-expression analyses, along with the comparison of mutant phenotypes, could help to limit the number of potential ROP-kinase pairs to be investigated.

Another Arabidopsis RLCK, belonging to the VIII subfamily and designated "NOVEL CYSTEINE-RICH KINASE”, is highly expressed during the differentiation of the tracheary elements. It also interacts with ROP GTPases [43]. However, the ROP-dependent activity of NOVEL CYSTEINE-RICH KINASE has not been experimentally verified. Nevertheless, the interaction raises the possibility that further RLCKs outside of the RLCK VI family may also serve as ROP effectors. The ROP-binding RLCK proteins do not have any known GTPasebinding motifs, and the structural requirements for ROP GTPase-mediated kinase activation in plants are currently unknown. The determination of the amino acid motifs that play a role in the ROP-binding of RLCK VI_A kinases could also help in the screening of the approximately 150 Arabidopsis RLCK sequences for further potential ROP-activated kinases.

\subsection{The SNF1-RELATED PROTEIN KINASE TYPE 2 kinase is indirectly regulated by}

\section{ROPs}

The active ROP11 GTPase can interact with the ABSCISIC ACID-INSENSITIVE 1 and ABSCISIC ACID-INSENSITIVE 2 PP2C-type phosphatases, negative regulators of abscisic acid signalling [21]. In the absence of abscisic acid, ABSCISIC ACID-INSENSITIVE 1/2 keeps SNF1-RELATED PROTEIN KINASE TYPE 2 protein kinases in an inactive dephosphorylated state, and the phosphorylation of downstream kinase targets is blocked. These targets include abscisic acid-regulated transcription factors, ion channels and ROS- 
producing enzymes (for a recent review, see [96]). The SNF1-RELATED PROTEIN KINASE TYPE 2 -phosphatase activity of ABSCISIC ACID-INSENSITIVE 2 is enhanced in the presence of the GTP-bound AtROP11 protein [21]. This interaction prevents the binding of the ABSCISIC ACID-INSENSITIVE 2 protein to its inhibitors: the abscisic acid receptor PYRABACTIN/PYRABACTIN-LIKE PROTEINs [55]. Therefore, the active AtROP11 GTPase negatively regulates the signalling downstream of the abscisic acid receptor. Although the SNF1-RELATED PROTEIN KINASE TYPE 2 kinase is not a ROP effector, the AtROP11 GTPase indirectly regulates its activity (Fig. 4).

The role of AtROP10 (and AtROP6) in the negative regulation of abscisic acid signalling has already been reported in Arabidopsis [97]; however, AtROP10 does not directly interact with ABSCISIC ACID-INSENSITIVE 2, and probably regulates other components of the abscisic acid response [21].

\subsection{ROP GTPases and the MITOGEN-ACTIVATED PROTEIN KINASE cascades}

Small Ras/Rho-type GTP-binding proteins frequently mediate the receptor-dependent activation of mitogen-activated protein kinase cascades in animal cells [98]. Knowledge about the role of ROPs - the only Ras/Rho-type small plant GTPases - in the regulation of MAPK cascades is rather scarce [80]. Although MAPKs are unlikely to serve as direct ROP GTPase effectors, ROPs and MAPKs can be present in the same signalling protein complex. For example, although OsMAPK6 and OsRAC1 (a rice ROP GTPase) do not directly interact in the yeast two-hybrid system, the constitutively active OsRAC1 could be specifically coimmunoprecipitated with OsMAPK6 from sphingolipid-elicited rice cells [81]. In addition, OsMAPK3 is part of the same OsRAC1-containing protein complex that regulates the RAC IMMUNITY 1 transcription factor via phosphorylation [99]. Neither dominant negative, nor non-isoprenylated mutant forms of OsRAC1 could form complexes with OsMAPK6. Moreover, the overexpression of these inactive and/or mis-localized mutant OsRAC1 forms 
prevents the sphingolipid elicitor-mediated activation of the kinase [81]. The RNAi-mediated silencing of OsRAC1 resulted in decreased OsMAPK6 protein (but not mRNA) levels and activity [81]. These observations indicate that the elicitor-triggered activation of OsMAPK6 takes place in a protein complex localized at the plasma membrane by GTP-bound and isoprenylated OsRAC1. This protein complex is not formed in the absence of OsRAC1 and the free MAPK is probably less stable, which can explain the reduced OsMAPK6 levels in OsRAC1 RNAi plants. The active G-protein may have an important role in the localization of the OsMAPK3/6-related signalling module to the plasma membrane, and more specifically, to the defined detergent-resistant microdomain raft regions $[80,100]$. Therefore, the OsRAC1 protein positively and indirectly regulates the MAPK-dependent signalling, through the promotion of protein complex formation at defined plasma membrane regions.

MAPK signalling cascades are implicated in many plant developmental and defence pathways, several of which are also regulated by ROP GTPases. Therefore, crosstalk between these signalling modules is very likely. As briefly discussed above (Section 2.8), MAPKs may serve as upstream regulators of ROP signalling, through the phosphorylation of RopGEFs or RopGAPs (see also Fig. 3). However, at present, our knowledge on the ways in which ROPs may regulate downstream MAPK-signalling is scarce (Fig. 4). The missing links may be the ROP-activated kinases (e.g., RLCK VI_A), like the P21-ACTIVATED KINASEs in animal and yeast cells, or the ROP-binding scaffold proteins with currently unknown downstream targets (for reviews, see [2-4]). ROPs may also activate MAPK cascades indirectly through NADPH oxidase-mediated $\mathrm{H}_{2} \mathrm{O}_{2}$ production, since several MAPKs are activated by $\mathrm{H}_{2} \mathrm{O}_{2}$ [101]. For example, the FERONIA receptor-signalling pathway can be indirectly linked to MAPK cascades through the ROP-mediated activation of NADPH oxidase-dependent $\mathrm{H}_{2} \mathrm{O}_{2}$ production (see Section 2.3 and Fig. 4).

\subsection{ROPs and lipid kinases}


Phosphatidylinositol monophosphate kinases, which synthesize phosphatidylinositol 4,5bisphosphate (PIP2), are well known effectors of Rho-type GTPases [1]. In growing pollen tubes, PIP2 preferentially accumulates in the subapical area of the plasma membrane, due to the subapical distribution and activity of the PHOSPHATIDYLINOSITOL 4-PHOSPHATE 5-KINASE (PIP5K) that generates PIP2 [102]. The inhibition of PIP2 degradation results in the loss of pollen tube polarity and tip swelling [103], indicating its important role in polarity establishment. The phosphatidylinositol 4-phosphate kinase activity was physically associated with a GTP-bound ROP GTPase (NtRAC5) purified from tobacco pollen tubes, supporting the view that ROPs might regulate PIP5K activity [102]. Through the stimulation of PIP2 synthesis, ROPs can control the targeted secretion by regulating actin organization and exocytotic membrane traffic during polar growth [102]. PIP5Ks might not only act downstream but also upstream of ROPs [104, 105]. In tobacco pollen tubes, the overexpression of PIP5K11 resulted in a similar tip swelling phenotype to that of NtRAC5. This could be counteracted by the co-expression of RhoGDI2 protein, a negative regulator of NtRAC5. It is hypothesized that the PIP5K11 inactivates the GDI protein, resulting in the local accumulation of GTP-bound NtRAC5 that regulates polarity [105]. Engineering of the PIP5K11, RhoGDI2 protein and NtRAC5 mutants, which are all functional but cannot interact with each other, could help to unravel the significance of this interaction network in pollen tube polarity.

\section{Conclusions and perspectives}

Accumulating evidence supports the view that Rho-type GTPases are central to a variety of receptor-mediated signalling pathways in plants, just as in other eukaryotes. However, due to the early separation of plants, fungi and metazoa during evolution, there are specific Rhotype GTPases (ROPs), as well as specific receptor kinase families, that have evolved in plants 
$[3,6]$. It has become evident that numerous plant receptor kinases, belonging to different families ([9]; also, see Sections 2.1-2.7 for details), use RopGEFs that transduce signals towards ROP GTPases, which can activate multiple effectors and induce a variety of cellular responses. The possible combination of the high number of receptor kinases (above 600 in Arabidopsis), with the numerous RopGEFs, ROPs, and ROP effectors, provide the cell with ample possibilities to process—-spatially as well as temporally—a variety of signals evoking specific but intricate cellular responses.

In a generalized model, a receptor-like kinase phosphorylates a RopGEF, releasing its auto-inhibition resulting in the accumulation of GTP-bound active ROPs at the plasma membrane, due to enhanced ROP nucleotide exchange. The potential phosphorylation sites on the C-terminal regions of Arabidopsis and rice GEFs are compared in Fig. 2. Some of these seem to be evolutionarily conserved between Arabidopsis and rice, such as the experimentally investigated phosphorylation sites of AtRopGEF12, AtRopGEF1 and OsRacGEF1 proteins. The S480 residue of AtRopGEF1 is conserved in OsRacGEF1, and the S549 residue of OsRacGEF1 is conserved in AtRopGEF1 (Fig. 2), but they are only phosphorylated in the Arabidopsis or the rice protein, respectively $[15,32]$. One possible explanation for this is that, while the phosphorylation of the Arabidopsis protein by the AtPOLLEN RECEPTOR KINASE 2 was investigated in the context of pollen tube growth [15], the phosphorylation of OsRacGEF1 by the CERK1 kinase was studied in relation to pathogen defence [32]. This hypothesis could be confirmed by investigating the functions and phosphorylation patterns of AtRopGEF1 during pathogen defence and OsRacGEF1 during polar growth. It is likely that the activity of GEFs could be fine-tuned, due to the phosphorylation of a number of serine/threonine residues, either alone or in combination with various kinases.

Experimental data on specific phosphorylation events are mostly indirect or lacking, and therefore, there is much room for further research on this subject. Moreover, kinase activity is 
not always necessary for receptor-like kinase-mediated downstream signalling towards ROPs, such as in the case of FERONIA-mediated pollen tube rupture in Arabidopsis [59] or PANGLOSS1-regulated stomatal patterning in maize leaves [31]. These kinases may serve as scaffolds for the assembly of large complexes, potentially including other kinases. The importance of a spatially regulated receptor complex formation is unquestionable in ROPmediated processes. ROPs preferentially accumulate in specific detergent-resistant membrane microdomains or lipid rafts (e.g., [100]), which serve as organizing centres for the assembly of signalling complexes in eukaryotic cells. In these regions, ROPs may promote the recruitment and/or assembly of receptor complex components, as well as downstream signalling elements.

The ROP regulators, as well as the ROPs themselves have many potential phosphorylation sites that can be targets of cytoplasmic as well as receptor kinases (Figs. 3 and 4). The phosphorylation of ROPs and their regulators allows the cell to fine-tune not only the ROPcentred signal transduction, but also the cytoskeletal dynamics. The potential of MITOGENACTIVATED PROTEIN KINASEs (MAPKs) to modulate ROP-mediated signal transduction is supported by the presence of MAPK docking and phosphorylation motifs on most RopGEF and on certain ROP GTPASE ACCELERATOR PROTEINs ([80], Fig. 2). The phosphorylation of RopGEFs by MAPKs, in addition to the receptor-like kinases, can serve as efficient means for signal integration. Future research identifying RopGEF-kinases and phosphorylation-dependent RopGEF functions will likely reveal the complexity of the phosphorylation networks centred on RopGEFs.

ROP G-proteins may also regulate downstream MAPK signalling cascades, either directly or indirectly, as described in relation to the OsRAC1-involving defence pathways of rice cells. ROPs may directly influence the subcellular location of MAPK-containing signalling complexes. This possibility is supported by the presence of MAPK-docking motifs on many 
ROPs [80]. Our present knowledge about ROP-regulated gene expression changes is rather limited. MAPK cascades may play a central role in the mediation of ROP-dependent signals towards transcription factors. MAPKs can also serve as candidates interlinking ROPdependent and independent signalling pathways within plant cells. Therefore, the link between ROP- and MAPK-mediated processes definitely deserves more attention in the future. Investigating MAPK activities in ROP signalling mutants, and vice versa, investigating ROP signalling in mutants defective in MAPK functions can give more insights into these processes.

PHOSPHATIDYLINOSITOL 4-PHOSPHATE 5-KINASEs (PIP5Ks) are another group of kinases that control ROP-mediated signalling both upstream and downstream of the small GTPases (Figs. 3 and 4). The details of these interactions could be revealed via targeted cellular, biochemical and genetic approaches (e.g., spatial and temporal resolution of proteinprotein interactions and localizations, kinase activity assays, mutant analyses, etc.), aiming to demonstrate the potential feedback regulation between phosphatidylinositol 4,5-bisphosphate synthesis and cell polarity establishment.

The CALCIUM-DEPENDENT PROTEIN KINASEs (CPKs) form a large group of intracellular kinases that may also have an intricate relationship with ROP GTPases. CPKs may phosphorylate upstream and downstream components that modulate the signalling functions of ROPs (Fig. 3). The relationship between ROP- and CPK-dependent signalling pathways is an area of research that has hardly been explored. Testing the CPK-dependent phosphorylation of ROP signalling components, followed by functional assays, would be required to underline this relationship experimentally.

The biological function of RECEPTOR-LIKE CYTOPLASMIC KINASEs (RLCKs), which are directly activated by ROP GTPases, also awaits exploration (Fig. 4). At present, we only know about a limited number of RLCKs (class VI group A) exhibiting some ROP- 
dependent activity [44-46]. The high number of RLCKs (approx. 200 in Arabidopsis) suggests further signalling interactions. Revealing the structural requirements for the ROPRLCK interaction could represent a big step forward, especially as this relies on as-yetunknown sequence motifs.

The link between ROP GTPases (the only signalling-type small GTPases in plants) and upstream as well as downstream signal transduction pathways (Figs. 3 and 4) remained in obscurity for a long time. The association of ROPs with receptor and cytoplasmic kinases has been recently demonstrated in a number of cases, shedding more light on their signalling power (Figs. 3 and 4). However, only further extensive research can reveal the ROP GTPasedependent signalling in plants in its full complexity. The common story of plant GTPases and kinases has barely begun.

\section{Acknowledgements}

The authors acknowledge the support of the Hungarian Scientific Research Fund (OTKA K101112). We apologize to those of our colleagues whose scientific work is not cited due to space restrictions.

\section{References}

[1] A. L. Bishop, A. Hall, Rho GTPases and their effector proteins, Biochem. J. 348 (2000) 241-255.

[2] A. Berken, A. Wittinghofer, Structure and function of Rho-type molecular switches in plants, Plant Physiol. Biochem. 46 (2008) 380-393.

[3] T. Brembu, P. Winge, A.M. Bones, Z. Yang, A RHOse by any other name: a comparative analysis of animal and plant Rho GTPases, Cell Res. 16 (2006) 435-445. 
[4] S. Nagawa, T. Xu, Z. Yang, RHO GTPase in plants: Conservation and invention of regulators and effectors, Small GTPases 1 (2010) 78-88.

[5] T. Christensen, Z. Vejlupkova, Y. Sharma, KM, Conserved subgroups and developmental regulation in the monocot rop gene family, Plant Physiol. 133 (2003) 1791-1808.

[6] S. Shiu, A. Bleecker, Expansion of the receptor-like kinase/Pelle gene family and receptor-like proteins in Arabidopsis, Plant Physiol. 132 (2003) 530-543.

[7] D. Urano, A.M. Jones, "Round up the usual suspects": a comment on nonexistent plant G protein-coupled receptors, Plant Physiol. 161 (2013) 1097-1102.

[8] J. Qiu, R. Jilk, M.D. Marks, D.B. Szymanski, The Arabidopsis SPIKE1 gene is required for normal cell shape control and tissue development, Plant Cell 14 (2002) 101-118.

[9] K.N. Miyawaki, Z. Yang, Extracellular signals and receptor-like kinases regulating ROP GTPases in plants, Front. Plant Sci. 5 (2014) 449.

[10] M.R. Schiller, Coupling receptor tyrosine kinases to Rho GTPases--GEFs what's the link, Cell. Signal. 18 (2006) 1834-1843.

[11] C. Hofmann, M. Shepelev, J. Chernoff, The genetics of PAK, J. Cell Sci. 117 (2004) $4343-4354$.

[12] J. Li, F.E. Tax, Receptor-like kinases: key regulators of plant development and defense, J. Integr. Plant Biol. 55 (2013) 1184-1187. 
[13] A.E. Trotochaud, T. Hao, G. Wu, Z. Yang, S.E. Clark, The CLAVATA1 receptor-like kinase requires CLAVATA3 for its assembly into a signaling complex that includes KAPP and a Rho-related protein, Plant Cell 11 (1999) 393-406.

[14] Y. Zhang, S. McCormick, A distinct mechanism regulating a pollen-specific guanine nucleotide exchange factor for the small GTPase Rop in Arabidopsis thaliana, Proc. Natl. Acad. Sci. USA 104 (2007) 18830-18835.

[15] F. Chang, Y. Gu, H. Ma, Z. Yang, AtPRK2 Promotes ROP1 activation via RopGEFs in the control of polarized pollen tube growth, Mol. Plant 6 (2013) 1187-1201.

[16] P. Kaothien, et al., Kinase partner protein interacts with the LePRK1 and LePRK2 receptor kinases and plays a role in polarized pollen tube growth, Plant J. 42 (2005) 492-503.

[17] G.-Q. Huang, et al., Arabidopsis RopGEF4 and RopGEF10 are important for FERONIA-mediated developmental but not environmental regulation of root hair growth, New Phytol. 200 (2013) 1089-1101.

[18] Q. Duan, D. Kita, C. Li, A.Y. Cheung, H.-M. Wu, FERONIA receptor-like kinase regulates RHO GTPase signaling of root hair development, Proc. Natl. Acad. Sci. USA 107 (2010) 17821-17826.

[19] Z. Li, D. Liu, ROPGEF1 and ROPGEF4 are functional regulators of ROP11 GTPase in ABA-mediated stomatal closure in Arabidopsis, FEBS Lett. 586 (2012) 1253-1258.

[20] Z. Li, J. Kang, N. Sui, D. Liu, ROP11 GTPase is a negative regulator of multiple ABA responses in Arabidopsis, J. Integr. Plant Biol. 54 (2012) 169-179. 
[21] F. Yu, et al., FERONIA receptor kinase pathway suppresses abscisic acid signaling in Arabidopsis by activating ABI2 phosphatase, Proc. Natl. Acad. Sci. USA 109 (2012) 14693-14698.

[22] Q. Duan, et al., Reactive oxygen species mediate pollen tube rupture to release sperm for fertilization in Arabidopsis, Nat. Commun. 5 (2014) 3129.

[23] F. Yu, et al., FERONIA receptor kinase controls seed size in Arabidopsis thaliana, Mol. Plant 7 (2014) 920-922.

[24] J.-B. Huang, et al., ROP3 GTPase contributes to polar auxin transport and auxin responses and is important for embryogenesis and seedling growth in Arabidopsis, Plant Cell 26 (2014) 3501-3518.

[25] M. Chen, et al., RopGEF7 regulates PLETHORA-dependent maintenance of the root stem cell niche in Arabidopsis, Plant Cell 23 (2011) 2880-2894.

[26] A. Boisson-Dernier, et al., ANXUR receptor-like kinases coordinate cell wall integrity with growth at the pollen tube tip via NADPH oxidases, PLoS Biol. 11 (2013) e1001719.

[27] Y. Zou, M. Aggarwal, W.-G. Zheng, H.-M. Wu, A. Y. Cheung, Receptor-like kinases as surface regulators for RAC/ROP-mediated pollen tube growth and interaction with the pistil, AoB Plants 2011 (2011) plr017.

[28] T. Xu, et al., Cell surface ABP1-TMK auxin-sensing complex activates ROP GTPase signaling, Science 343 (2014) 1025-1028. 
[29] D. Lin, et al., A ROP GTPase-dependent auxin signaling pathway regulates the subcellular distribution of PIN2 in Arabidopsis roots, Curr. Biol. 22 (2012) 1319-1325.

[30] Y. Oda, H. Fukuda, Emerging roles of small GTPases in secondary cell wall development, Front. Plant Sci. 5 (2014) 428.

[31] J.A. Humphries, et al., ROP GTPases act with the receptor-like protein PAN1 to polarize asymmetric cell division in maize, Plant Cell 23 (2011) 2273-2284.

[32] A. Akamatsu, et al., An OsCEBiP/OsCERK1-OsRacGEF1-OsRac1 module is an essential early component of chitin-induced rice immunity, Cell Host Microbe. 13 (2013) 465-476.

[33] D. Ke, et al., Small GTPase ROP6 interacts with NFR5 and is involved in nodule formation in Lotus japonicus, Plant Physiol. 159 (2012) 131-143.

[34] S. Betsuyaku, et al., Mitogen-activated protein kinase regulated by the CLAVATA receptors contributes to shoot apical meristem homeostasis, Plant Cell Physiol. 52 (2011) 14-29.

[35] A. Fehér, M.E. Jurca, C. Fodor-Dunai, D. Dorjgotov, Regulation of ROP GTPase signalling at the gene expression level: a review, Open Plant Sci. J. 2 (2008) 21-30.

[36] D. Wengier, et al., The receptor kinases LePRK1 and LePRK2 associate in pollen and when expressed in yeast, but dissociate in the presence of style extract, Proc. Natl. Acad. Sci. USA 100 (2003) 6860-6865.

[37] Y. Zhang, S. McCormick, Regulation of pollen tube polarity, Plant Signal. Behav. 3 (2008) 345-347. 
[38] A. Berken, C. Thomas, A. Wittinghofer, A new family of RhoGEFs activates the Rop molecular switch in plants, Nature 436 (2005) 1176-1180.

[39] Y. Gu, S. Li, E.M. Lord, Z. Yang, Members of a novel class of Arabidopsis Rho guanine nucleotide exchange factors control Rho GTPase-dependent polar growth, Plant Cell 18 (2006) 366-381.

[40] S. Löcke, I. Fricke, E. Mucha, M.-L. Humpert, A. Berken, Interactions in the pollenspecific receptor-like kinases-containing signaling network, Eur. J. Cell Biol. 89 (2010) 917-923.

[41] A. Bellucci, C. Fiorentini, M. Zaltieri, C. Missale, P. Spano, The "in situ" proximity ligation assay to probe protein-protein interactions in intact tissues, Methods Mol. Biol. 1174 (2014) 397-405.

[42] Y. Qin, Z. Yang, Rapid tip growth: insights from pollen tubes, Semin. Cell Dev. Biol. 22 (2011) 816-824.

[43] A.J. Molendijk, et al., A cysteine-rich receptor-like kinase NCRK and a pathogeninduced protein kinase RBK1 are Rop GTPase interactors, Plant J. 53 (2008) 909-923.

[44] D. Dorjgotov, et al., Plant Rho-type (Rop) GTPase-dependent activation of receptorlike cytoplasmic kinases in vitro, FEBS Lett. 583 (2009) 1175-1182.

[45] C. Huesmann, et al., Barley ROP binding kinase1 is involved in microtubule organization and in basal penetration resistance to the barley powdery mildew fungus, Plant Physiol. 159 (2012) 311-320. 
[46] T. Reiner, et al., The Arabidopsis ROP-activated receptor-like cytoplasmic kinase RLCK VI_A3 is involved in control of basal resistance to powdery mildew and trichome branching, Plant Cell Rep. 34 (2014) 457-468.

[47] S. Okuda, T. Higashiyama, Pollen tube guidance by attractant molecules : LUREs signaling in sporophytic guidance, Cell Struct. Funct. 52 (2010) 45-52.

[48] M.A. Jones, M.J. Raymond, Z. Yang, N. Smirnoff, NADPH oxidase-dependent reactive oxygen species formation required for root hair growth depends on ROP GTPase, J. Exp. Bot. 58 (2007) 1261-1270.

[49] J. Foreman, et al., Reactive oxygen species produced by NADPH oxidase regulate plant cell growth, Nature 422 (2003) 442-446.

[50] E. Himschoot, T. Beeckman, J. Friml, S. Vanneste, Calcium is an organizer of cell polarity in plants, Biochim. Biophys. Acta - Mol. Cell Res. (2015). doi:10.1016/j.bbamcr.2015.02.017.

[51] M. Haruta, G. Sabat, K. Stecker, B.B. Minkoff, M.R. Sussman, A peptide hormone and its receptor, Science 343 (2014) 408-411.

[52] T.N. Bibikova, T. Jacob, I. Dahse, S. Gilroy, Localized changes in apoplastic and cytoplasmic $\mathrm{pH}$ are associated with root hair development in Arabidopsis thaliana, Development 125 (1998) 2925-2934.

[53] J. Wu, et al., NaRALF, a peptide signal essential for the regulation of root hair tip apoplastic $\mathrm{pH}$ in Nicotiana attenuata, is required for root hair development and plant growth in native soils, Plant J. 52 (2007) 877-890. 
[54] S. Wolf, H. Höfte, Growth control: A saga of cell walls, ROS, and peptide receptors, Plant Cell 26 (2014) 1848-1856.

[55] Z. Li, et al., ROP11 GTPase negatively regulates ABA signaling by protecting ABI1 phosphatase activity from inhibition by the ABA receptor RCAR1/PYL9 in Arabidopsis, J. Integr. Plant Biol. 54 (2012) 180-188.

[56] C. Nibau, L. Tao, K. Levasseur, H.-M. Wu, A.Y. Cheung, The Arabidopsis small GTPase AtRAC7/ROP9 is a modulator of auxin and abscisic acid signalling, J. Exp. Bot. 64 (2013) 3425-3437.

[57] J.-M. Escobar-Restrepo, et al., The FERONIA receptor-like kinase mediates malefemale interactions during pollen tube reception, Science 317 (2007) 656-660.

[58] M.M. Kanaoka, K.U. Torii, FERONIA as an upstream receptor kinase for polar cell growth in plants, Proc. Natl. Acad. Sci. USA 107 (2010) 17461-17462.

[59] S.A. Kessler, H. Lindner, D.S. Jones, U. Grossniklaus, Functional analysis of related CrRLK1L receptor-like kinases in pollen tube reception, EMBO Rep. (2014) 1-9.

[60] M. Potocký, et al., NADPH oxidase activity in pollen tubes is affected by calcium ions, signaling phospholipids and Rac/Rop GTPases, J. Plant Physiol. 169 (2012) 16541663.

[61] D. Zhang, et al., The pollen receptor kinase LePRK2 mediates growth-promoting signals and positively regulates pollen germination and tube growth, Plant Physiol. 148 (2008) 1368-1379. 
[62] S. Li, Y. Zhang, To grow or not to grow: FERONIA has her say, Mol. Plant 7 (2014) $1261-1263$.

[63] A.Y. Cheung, H.-M. Wu, THESEUS 1, FERONIA and relatives: a family of cell wallsensing receptor kinases? Curr. Opin. Plant Biol. 14 (2011) 632-641.

[64] P. Qian, S. Hou, G. Guo, Molecular mechanisms controlling pavement cell shape in Arabidopsis leaves, Plant Cell Rep. 28 (2009) 1147-1157.

[65] T. Xu, et al., Cell surface-and Rho GTPase-based auxin signaling controls cellular interdigitation in Arabidopsis, Cell 143 (2010) 99-110.

[66] G.F.E. Scherer, AUXIN-BINDING-PROTEIN1, the second auxin receptor: What is the significance of a two-receptor concept in plant signal transduction? J. Exp. Bot. 62 (2011) 3339-3357.

[67] Y. Gao, et al., Auxin binding protein 1 (ABP1) is not required for either auxin signaling or Arabidopsis development, Proc. Natl. Acad. Sci. USA 112 (2015) 22752280 .

[68] C. Zhang, S.O. Kotchoni, A. L. Samuels, D.B. Szymanski, SPIKE1 signals originate from and assemble specialized domains of the endoplasmic reticulum, Curr. Biol. 20 (2010) 2144-2149.

[69] H.N. Cartwright, J.A. Humphries, L.G. Smith, PAN1: a receptor-like protein that promotes polarization of an asymmetric cell division in maize, Science 323 (2009) $649-651$. 
[70] E. Ono, et al., Essential role of the small GTPase Rac in disease resistance of rice, Proc. Natl. Acad. Sci. USA 98 (2001) 759-764.

[71] Y. Kawano, L. Chen, K. Shimamoto, The function of Rac small GTPase and associated proteins in rice innate immunity, Rice 3 (2010) 112-121.

[72] Y. Kawano, K. Shimamoto, Early signaling network in rice PRR-mediated and Rmediated immunity, Curr. Opin. Plant Biol. 16 (2013) 496-504.

[73] A.A. Gust, R. Willmann, Y. Desaki, H.M. Grabherr, T. Nürnberger, Plant LysM proteins: modules mediating symbiosis and immunity, Trends Plant Sci. 17 (2012) 495-502.

[74] Y. Oda, H. Fukuda, Initiation of cell wall pattern by a Rho- and microtubule-driven symmetry breaking, Science 337 (2012) 1333-1336.

[75] G. Loirand, C. Guilluy, P. Pacaud, Regulation of Rho proteins by phosphorylation in the cardiovascular system, Trends Cardiovasc. Med. 16 (2006) 199-204.

[76] C. Fodor-Dunai, et al., The phosphomimetic mutation of an evolutionarily conserved serine residue affects the signaling properties of Rho of plants (ROPs), Plant J. 66 (2011) 669-679.

[77] I. Fricke, A. Berken, Molecular basis for the substrate specificity of plant guanine nucleotide exchange factors for ROP, FEBS Lett. 583 (2009) 75-80.

[78] T. Kwon, D.Y. Kwon, J. Chun, J.H. Kim, S.S. Kang, Akt protein kinase inhibits Rac1GTP binding through phosphorylation at serine 71 of Rac1, J. Biol. Chem. 275 (2000) 423-428. 
[79] E.H. Rademacher, R. Offringa, Evolutionary adaptations of plant AGC kinases: from light signaling to cell polarity regulation, Front. Plant Sci. 3 (2012) 250.

[80] O. Šamajová, G. Komis, J. Šamaj, Emerging topics in the cell biology of mitogenactivated protein kinases, Trends Plant Sci. 18 (2013) 140-148.

[81] D. Lieberherr, et al., A Sphingolipid elicitor-inducible mitogen-activated protein kinase is regulated by the small GTPase OsRac1 and heterotrimeric G-Protein in rice, Plant Physiol. 138 (2005) 1644-1652.

[82] J. Hwang, V. Vernoud, A. Szumlanski, A tip-localized RhoGAP controls cell polarity by globally inhibiting Rho GTPase at the cell apex, Curr. Biol. 18 (2008) 1907-1916.

[83] A. Jamin, Z. Yang, Interactions between calcium and ROP signaling regulate pollen tube tip growth, in: S. Luan (Ed.), Coding and Decoding Calcium Signals in Plants, Springer Berlin Heidelberg, Berlin, Heidelberg, 2011: pp. 25-39.

[84] C. Myers, et al., Calcium-dependent protein kinases regulate polarized tip growth in pollen tubes, Plant J. 59 (2009) 528-539.

[85] Y. Wu, et al., CPK3-phosphorylated RhoGDI1 is essential in the development of Arabidopsis seedlings and leaf epidermal cells, J. Exp. Bot. (2013) 1-18.

[86] J. Fíla, et al., Revealing phosphoproteins playing role in tobacco pollen activated in vitro, Proteomics 12 (2012) 3229-3250.

[87] Y. Guan, J. Guo, H. Li, Z. Yang, Signaling in pollen tube growth: crosstalk, feedback, and missing links, Mol. Plant 6 (2013) 1053-1064. 
[88] J.-U. Hwang, Y. Gu, Y.-J. Lee, Z. Yang, Oscillatory ROP GTPase activation leads the oscillatory polarized growth of pollen tubes, Mol. Biol. Cell 16 (2005) 5385-5399.

[89] G. Wu, Y. Gu, S. Li, Z. Yang, A genome-wide analysis of Arabidopsis Rop-Interactive CRIB Motif-Containing Proteins that act as Rop GTPase targets, Plant Cell 13 (2001) $2841-2856$.

[90] S.W. Hsu, C.S. Wang, Lily Cdc42/Rac-interactive binding motif-containing protein, a Rop target, involves calcium influx and phosphoproteins during pollen germination and tube growth, Plant Signal. Behav. 5 (2010) 1460-1463.

[91] W. Lin, X. Ma, L. Shan, P. He, Big roles of small kinases: the complex functions of receptor-like cytoplasmic kinases in plant immunity and development, J. Integr. Plant Biol. 55 (2013) 1188-1197.

[92] M.E. Jurca, S. Bottka, A. Fehér, Characterization of a family of Arabidopsis receptorlike cytoplasmic kinases (RLCK class VI), Plant Cell Rep. 27 (2008) 739-748.

[93] H. Schultheiss, C. Dechert, K.-H. Kogel, R. Hückelhoven, Functional analysis of barley RAC/ROP G-Protein family members in susceptibility to the powdery mildew fungus, Plant J. 36 (2003) 589-601.

[94] J.D. Becker, S. Takeda, F. Borges, L. Dolan, J. A Feijó, Transcriptional profiling of Arabidopsis root hairs and pollen defines an apical cell growth signature, BMC Plant Biol. 14 (2014) 197.

[95] H. Li, J. Shen, Z.-L. Zheng, Y. Lin, Z. Yang, The Rop GTPase switch controls multiple developmental processes in Arabidopsis, Plant Physiol. 126 (2001) 670-684. 
[96] K. Nakashima, K. Yamaguchi-Shinozaki, ABA signaling in stress-response and seed development, Plant Cell Rep. 32 (2013) 959-970.

[97] Z.L. Zheng, et al., Plasma membrane-associated ROP10 small GTPase is a specific negative regulator of abscisic acid responses in Arabidopsis, Plant Cell 14 (2002) 2787-2797.

[98] A. Vojtek, J. Cooper, Rho family members: activators of MAP kinase cascades, Cell 82 (1995) 527-529.

[99] S.-H. Kim, et al., The bHLH Rac Immunity1 (RAI1) is activated by OsRac1 via OsMAPK3 and OsMAPK6 in rice immunity, Plant Cell Physiol. 53 (2012) 740-754.

[100] M. Fujiwara, et al., Proteome analysis of detergent-resistant membranes (DRMs) associated with OsRac1-mediated innate immunity in rice, Plant Cell Physiol. 50 (2009) 1191-1200.

[101] S. Hung, C. Yu, C. Lin, Hydrogen peroxide functions as a stress signal in plants, Bot Bull Acad Sin. 46 (2005) 1-10.

[102] B. Kost, et al., Rac homologues and compartmentalized phosphatidylinositol 4, 5bisphosphate act in a common pathway to regulate polar pollen tube growth, J. Cell Biol. 145 (1999) 317-330.

[103] D. Helling, A. Possart, S. Cottier, U. Klahre, B. Kost, Pollen tube tip growth depends on plasma membrane polarization mediated by tobacco PLC3 activity and endocytic membrane recycling, Plant Cell 18 (2006) 3519-3534. 
[104] B. Kost, Spatial control of Rho (Rac-Rop) signaling in tip-growing plant cells, Trends Cell Biol. 18 (2008) 119-127.

[105] T. Ischebeck, et al., Phosphatidylinositol-4,5-bisphosphate influences Nt-Rac5mediated cell expansion in pollen tubes of Nicotiana tabacum, Plant J. 65 (2011) 453468.

\section{Figure legends}

Figure 1. Rho G-proteins serve as two-state molecular switches. They are "ON" in the GTP-bound conformation when they can bind and activate (indicated by an asterisk) various effector molecules, evoking specific cellular responses. Hydrolysis of the bound GTP results in the "OFF" state, when the effectors are released and/or inactive. GDP-to-GTP exchange can revert the protein back to the "ON" state. Three main types of proteins regulate the switch spatially and temporally: the guanine nucleotide exchange factors (GEFs) enhancing the GDP-to-GTP exchange, switching the G-protein "ON"; GTPase accelerating proteins (GAPs) promoting GTP-hydrolysis that switches the protein "OFF"; and the guanine nucleotide dissociation inhibitors (GDIs) temporarily stabilizing the "OFF" state.

Figure 2. Potential phosphorylation sites of C-terminal RopGEF regions. The alignment of the C-terminal regions of two groups (A and B) of homologous Arabidopsis and rice RopGEFs is shown (a third group consisting of Os10g0550300, Os04g47170, Os07g0481100, AtRopGEF2, AtRopGEF3 and AtRopGEF4 has no similar C-terminal domains, and therefore is not included in the figure). The same letter colour is used to label amino acids that have similar biochemical characters. Black boxes indicate the potential MAPK phosphorylation 
sites (determined in silico, using the Group-based Prediction System v3.0,

http://gps.biocuckoo.org). Note that while a number of them are evolutionarily conserved, many others are protein-specific. The serine residues serving as potential receptor-like kinase target sites, based on experimental data (see Section 2.2 for details), are circled in red. Note that the S510 site of AtRopGEF12 is a potential MAPK as well as receptor-like kinase site and it is widely conserved. The other two potential receptor-like kinase target sites are conserved between the closely homologous AtRopGEF1 and OsRacGEF1 proteins, but appear in hardly any other RopGEFs.

Figure 3. Schematic summary of the present knowledge on potential direct/indirect interactions of ROP GTPases with upstream regulatory kinases, as detailed in the text. Green shapes represent the kinases. Arrows indicate signalling interaction (activation or inhibition), which does not necessarily mean phosphorylation. The interactions illustrated together on the figure are temporally and spatially separated within the cell. Most of the proteins are members of various protein families, which gives them specificity while at the same time increasing the complexity of regulations. To preserve simplicity, not all possible interactions are shown among the presented molecules.

AGC? - POTENTIAL AGC-TYPE KINASE; CPK - CALCIUM-DEPENDENT PROTEIN KINASE; GAP - GTPASE ACCELERATING PROTEIN; GDI - GUANINE NUCLEOTIDE DISSOCIATION INHIBITOR; GEF - GUANINE NUCLEOTIDE EXCHANGE FACTOR; MITOGEN ACTIVATED PROTEIN KINASE - MITOGEN ACTIVATED PROTEIN KINASE; PIP5K - PHOSPHATIDYLINOSITOL 4-PHOSPHATE 5-KINASE; RLK - RECEPTOR-LIKE KINASE; ROP - RHO OF PLANTS 
Figure 4. Schematic summary of the present knowledge on potential direct/indirect interactions of ROP GTPases with downstream signal-transducing kinases, as detailed in the text. Green shapes represent the kinases. Arrows indicate signalling interaction (activation or inhibition), which does not necessarily mean the regulation of phosphorylation activity. The interactions illustrated together on the figure are temporally and spatially separated within the cell. Most of the proteins are members of various protein families, which gives them specificity while at the same time increasing the complexity of regulations. To preserve simplicity, not all possible interactions are shown among the presented molecules.

ABI1/2 - ABSCISIC ACID-INSENSITIVE 1/2 PHOSPHATASE; NADPH OXIDASE NICOTINAMIDE ADENINE DINUCLEOTIDE PHOSPHATE-OXIDASE; PIP5K PHOSPHATIDYLINOSITOL 4-PHOSPHATE 5-KINASE; RLCK VI_A - RECEPTORLIKE CYTOPLASMIC KINASE CLASS VI GROUP A; ROP - RHO OF PLANTS; SNRK2 - SNF1-RELATED KINASE TYPE 2 
Table 1. List of the main protein families mentioned in the text

\begin{tabular}{|c|c|c|c|c|}
\hline Name & Abbreviation & Molecular function & Plants & $\begin{array}{l}\text { Yeast } \\
\text { and/c } \\
\text { Meta }\end{array}$ \\
\hline \multicolumn{5}{|c|}{ SMALL G-PROTEINS } \\
\hline RHO OF PLANTS & ROP & $\begin{array}{l}\text { small GTP-binding protein with } \\
\text { GTPase activity, RHO-type }\end{array}$ & YES & NO \\
\hline CELL DIVISION CYCLE 42 & Cdc42 & $\begin{array}{l}\text { small GTP-binding protein with } \\
\text { GTPase activity, RHO-type }\end{array}$ & NO & YES \\
\hline $\begin{array}{l}\text { RAS-RELATED C3 } \\
\text { BOTULINUM TOXIN } \\
\text { SUBSTRATE } 1\end{array}$ & Rac1 & $\begin{array}{l}\text { small GTP-binding protein with } \\
\text { GTPase activity, RHO-type }\end{array}$ & NO & YES \\
\hline RAS-HOMOLOG & Rho & $\begin{array}{l}\text { small GTP-binding protein with } \\
\text { GTPase activity, RHO-type }\end{array}$ & NO & YES \\
\hline RAT SARCOMA & Ras & $\begin{array}{l}\text { small GTP-binding protein with } \\
\text { GTPase activity, RAS-type }\end{array}$ & NO & YES \\
\hline \multicolumn{5}{|c|}{ UPSTREAM OF REVIEWED SMALL G-PROTEINS } \\
\hline $\begin{array}{l}\text { GUANINE NUCLEOTIDE } \\
\text { EXCHANGE FACTOR }\end{array}$ & GEF & $\begin{array}{l}\text { promotes the GDP-to-GTP } \\
\text { exchange and thus activates } \\
\text { RHO-type small G-proteins, } \\
\text { might have various catalytic } \\
\text { domains as listed below }\end{array}$ & & \\
\hline $\begin{array}{l}\text { with DBL-homology } \\
\text { domain }\end{array}$ & $\mathrm{DH}$ & & NO & YES \\
\hline $\begin{array}{l}\text { with Dedicator of } \\
\text { cytokinesis } 180 \text { domain }\end{array}$ & DOCK180 & & YES & YES \\
\hline $\begin{array}{l}\text { with Plant ROP } \\
\text { nucleotide exchanger } \\
\text { domain }\end{array}$ & PRONE & & YES & NO \\
\hline $\begin{array}{l}\text { GTPASE ACCELERATOR } \\
\text { PROTEIN }\end{array}$ & GAP & $\begin{array}{l}\text { promotes the GTP-hydrolysis by } \\
\text { the G-protein inactivating its } \\
\text { signalling function }\end{array}$ & YES & YES \\
\hline $\begin{array}{l}\text { GUANINE NUCLEOTIDE } \\
\text { DISSOCIATION INHIBITOR }\end{array}$ & GDI & $\begin{array}{l}\text { regulates membrane association } \\
\text { and blocks spontaneous } \\
\text { activation of the G-protein }\end{array}$ & YES & YES \\
\hline RECEPTOR-LIKE KINASE & RLK & $\begin{array}{l}\text { transmembrane receptor } \\
\text { serine/threonine kinase }\end{array}$ & YES & NO \\
\hline RECEPTOR TYROSINE & RTK & transmembrane receptor & NO & YES \\
\hline
\end{tabular}




\begin{tabular}{|c|c|c|c|c|}
\hline \multicolumn{2}{|l|}{ KINASE } & \multicolumn{3}{|l|}{ tyrosine kinase } \\
\hline $\begin{array}{l}\text { G-PROTEIN COUPLED } \\
\text { RECEPTOR }\end{array}$ & GPCR & $\begin{array}{l}\text { transmembrane receptor with } \\
\text { heterotrimeric G-protein } \\
\text { guanine nucleotide exchanger } \\
\text { (GEF) activity }\end{array}$ & NO? & YES \\
\hline $\begin{array}{l}\text { CALCIUM-DEPENDENT } \\
\text { PROTEIN KINASES }\end{array}$ & CPK & $\begin{array}{l}\text { transduction of } \mathrm{Ca}^{2+} \text { signal via } \\
\text { protein phosphorylation in } \\
\text { association with stress } \\
\text { responses and development }\end{array}$ & YES & NO \\
\hline $\begin{array}{l}\text { MITOGEN-ACTIVATED } \\
\text { PROTEIN KINASES }\end{array}$ & MAPK & $\begin{array}{l}\text { members of intracellular kinase } \\
\text { cascades transducing protein } \\
\text { phosphorylation signals; activate } \\
\text { gene transcription in response } \\
\text { to a variety of signals, and only } \\
\text { certain members are indeed } \\
\text { mitogen-activated }\end{array}$ & YES & YES \\
\hline \multicolumn{5}{|c|}{ DOWNSTREAM OF REVIEWED SMALL G-PROTEINS } \\
\hline $\begin{array}{l}\text { PLASMA MEMBRANE } \\
\text { NICOTINAMIDE ADENINE } \\
\text { DINUCLEOTIDE } \\
\text { PHOSPHATE-OXIDASE }\end{array}$ & $\begin{array}{l}\text { plasma } \\
\text { membrane } \\
\text { NADPH } \\
\text { oxidase }\end{array}$ & $\begin{array}{l}\text { a membrane- } \\
\text { bound enzyme complex that } \\
\text { generates superoxide (that is } \\
\text { quickly converted to hydrogen } \\
\text { peroxide) }\end{array}$ & YES & YES \\
\hline P21-ACTIVATED KINASE & PAK & $\begin{array}{l}\text { Cdc42/Rac1-activated protein } \\
\text { kinase with the CDC42/RAC1 } \\
\text { interactive binding (CRIB) } \\
\text { domain }\end{array}$ & NO & YES \\
\hline $\begin{array}{l}\text { RECEPTOR-LIKE } \\
\text { CYTOPLASMIC KINASE } \\
\text { CLASS VI GROUP A }\end{array}$ & RLCK VI_A & $\begin{array}{l}\text { ROP-activated cytoplasmic } \\
\text { protein kinase }\end{array}$ & YES & NO \\
\hline $\begin{array}{l}\text { ROP-INTERACTIVE CRIB } \\
\text { MOTIF-CONTAINING } \\
\text { PROTEINS }\end{array}$ & RIC & $\begin{array}{l}\text { ROP }^{\text {GTP }} \text {-interacting scaffold } \\
\text { proteins with } \mathrm{Cdc} 42 / \text { Rac1 } \\
\text { interactive binding (CRIB) motif }\end{array}$ & YES & NO \\
\hline $\begin{array}{l}\text { ABSCISIC ACID- } \\
\text { INSENSITIVE } 1 \text { AND } 2 \\
\text { PHOSPHATASE }\end{array}$ & $A B I 1 / 2$ & $\begin{array}{l}\text { protein phosphatases, negative } \\
\text { regulators of abscisic acid } \\
\text { signalling, dephosphorylate the } \\
\text { SnRK2 kinase }\end{array}$ & YES & YES \\
\hline $\begin{array}{l}\text { SNF1-RELATED KINASE } \\
\text { TYPE } 2\end{array}$ & SnRK2 & $\begin{array}{l}\text { cellular protein kinase involved } \\
\text { in abscisic acid signalling }\end{array}$ & YES & YES \\
\hline $\begin{array}{l}\text { MITOGEN-ACTIVATED } \\
\text { PROTEIN KINASES }\end{array}$ & MAPK & $\begin{array}{l}\text { members of intracellular kinase } \\
\text { cascades transducing protein } \\
\text { phosphorylation signals; activate }\end{array}$ & YES & YES \\
\hline
\end{tabular}


gene transcription in response

to a variety of signals, and only

certain members are indeed

mitogen-activated

PHOSPHATIDYLINOSITOL PIP5K

lipid kinase responsible for the

YES

YES 4-PHOSPHATE 5-KINASE

production of

phosphatidylinositol 4, 5-

bisphosphate (PIP2)

The list of upstream regulators and downstream effectors of RHO-type GTPases included in the table are not complete, only those proteins are listed which are mentioned in the text. For a more detailed list, see the reviews [1-4]. YES; NO - the given proteins or their close structural homologs are present in PLANTS or YEASTS and/or METAZOA or not.; ? - indicates controversy 
Table 2. Reported and potential RLK-ROP GEF-ROP receptor signalling modules

\begin{tabular}{|c|c|c|c|c|c|}
\hline RLK & GEF & ROP & process & species & reference \\
\hline CLV1 & RopGEF? & ROP? & shoot meristem maintenance & At & [13] \\
\hline PRK2 & RopGEF12 & $\mathrm{ROP} 1 / 3 / 5 ?$ & pollen tube growth & At & {$[14]$} \\
\hline PRK2 & RopGEF1 & ROP1 & pollen tube growth & At & {$[15]$} \\
\hline LePRK1; 2 & KPP & ROP? & pollen tube growth & Le & {$[16]$} \\
\hline FERONIA & RopGEF1; 4; 10 & ROP2; 6 & root hair growth, auxin response & At & {$[17,18]$} \\
\hline FERONIA & RopGEF1; 4; 10 & AtROP11 & response to abscisic acid & At & [19-21] \\
\hline FERONIA & RopGEF? & ROP? & pollen tube rupture & At & {$[22]$} \\
\hline FERONIA & RopGEF1; ? & ROP? & seed size control & At & {$[23]$} \\
\hline FERONIA? & RopGEF7 & ROP3 & $\begin{array}{l}\text { auxin responses, root meristem } \\
\text { maintenance }\end{array}$ & At & {$[24,25]$} \\
\hline ANXURE1; 2 & RopGEF? & ROP? & pollen tube integrity & At & {$[26,27]$} \\
\hline TMK1-4 & SPIKE1 & ROP2; $4 ; 6$ & $\begin{array}{l}\text { auxin-mediated epidermal cell } \\
\text { morphology }\end{array}$ & At & {$[28]$} \\
\hline TMK? & SPIKE1 & ROP6 & $\begin{array}{l}\text { auxin signalling and transport in } \\
\text { the root }\end{array}$ & At & [29] \\
\hline$?$ & RopGEF4 & ROP11 & secondary cell wall patterning & At & {$[30]$} \\
\hline PAN1 & RopGEF? & ROP2; 9 & stomatal complex patterning & $\mathrm{Zm}$ & {$[31]$} \\
\hline CERK1 & RACGEF1 & RAC1 & pathogen defence signalling & Os & {$[32]$} \\
\hline NFR5 & RopGEF? & ROP6 & symbiotic interaction & $\mathrm{Lj}$ & [33] \\
\hline
\end{tabular}

RLK - receptor-like kinase; GEF - guanine nucleotide exchange factor; ROP - RHO-OF-PLANTS;

CLV1 -CLAVATA1; PRK - POLLEN RECEPTOR KINASE; TMK - TRANSMEMBRANE KINASE; PAN1 -PANGLOSS1; CERK1 - CHITIN ELICITOR RECEPTOR KINASE1; NFR5 - NOD FACTOR RECEPTOR5;

At - Arabidopsis thaliana; $\mathrm{Zm}$ - Zea mays; Os - Oryza sativa $; \mathrm{Lj}$ - Lotus japonicas

? - indicates the missing or ambiguous information on the given protein of the potential module. 


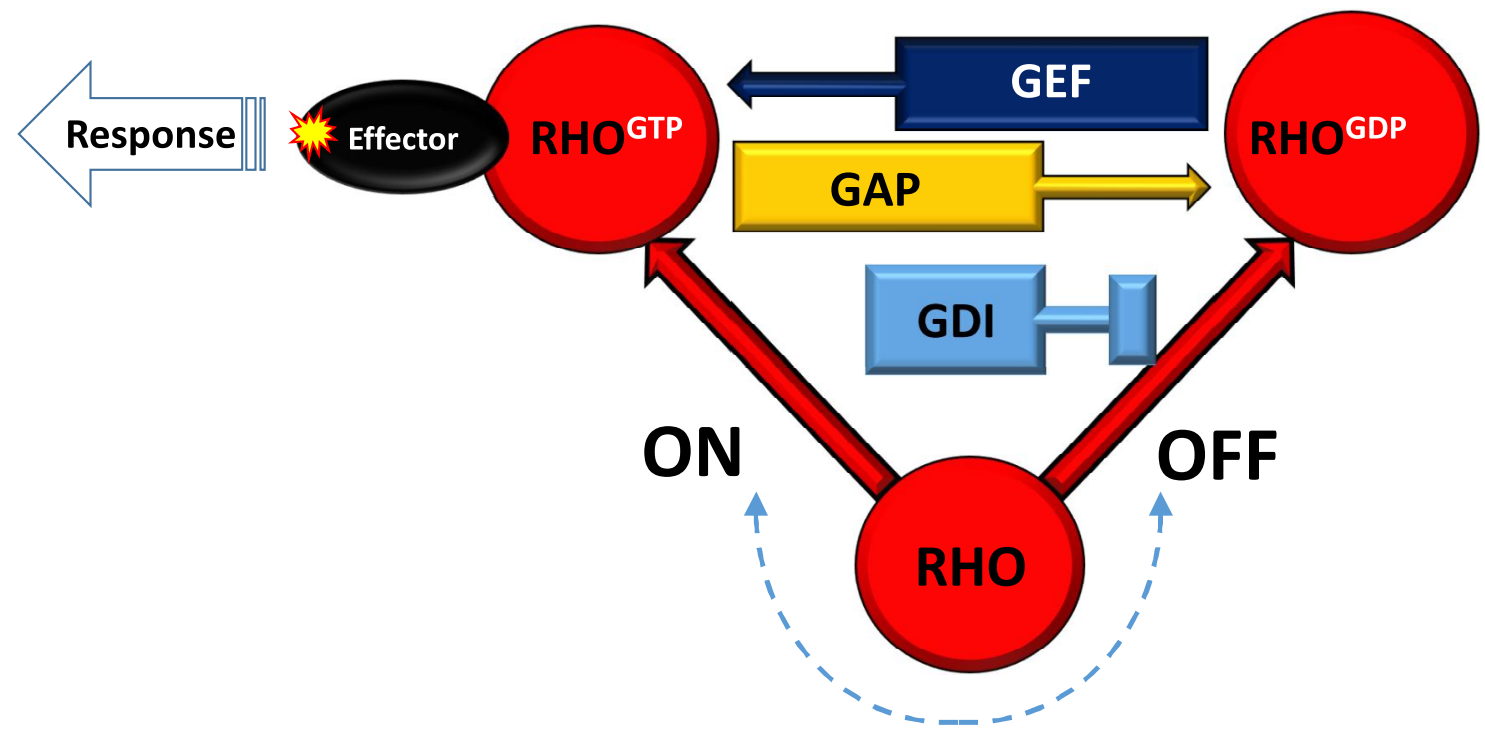




\section{A}

ATROPGEF8 ATROPGEF9 ATROPGEF10 ATROPGEF12 Os02g0272300 Os01g0760300 Os05g0560100 Os01g067500 ATROPGEF11 ATROPGEF13 Os02g070260 ATROPGEF14

DTLALKQTLLAEETS-DGGRTT--ETDSESAGSSNS-----GEEA----EKHDPHSKTLIDFMG-WNDNSSK----------------GG-DKPTKS

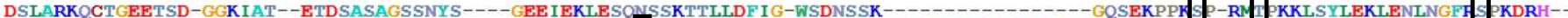
DRLVORO DAAAQNLTATEAARR-LMDGDA--------AARKLDA-----MEELEKLNEAPASMTLYDFMG-WHFDQDE-----LMRKKEEGTLDEAGEAKLKKAP-SLAPKKFSYVDSL-SAGGMP S SARH--

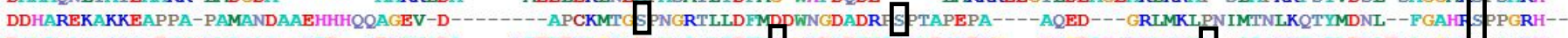
DSLAQASNTRTQESM-RMASLS--RYD--TDKVVIDA----KAEVERLGRMEPVSATLFDFVE PRDQDVVATKMDSKEKG---CRGDAHSRKLTKVE PIATKRYSYLEKL.ENLSGTHS ISRH--

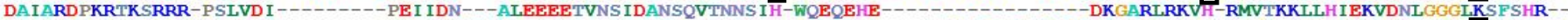
DOI DQLTQNPELAMCKIH-IVKETE------ SPEKFEFP--NFCLLEDRPKKOKPTISLSEVMQ-WN IETNE-----PRKEK----SDKKLLTRVSSMI -MSNNKKTTYLES---LGTTASPTAGRYS

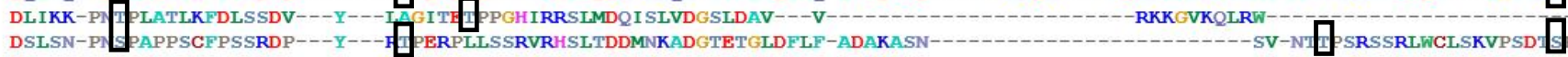

\section{B}

Os01g62990 VPPYQV---PAS TPFVT---AYATPSF-------SPAQL/SSSKKE---------RSLGAGR------RS--YSNRGFGAKKALAIDLVNPEVMGVI

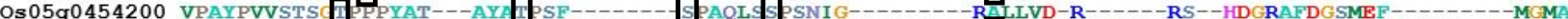
ATROPGEF5 VPLPYL--ISAS TPYRTS--FST PGP------- S SSM-IS FKKGE-----R---R PYSSKDTNKI I EKG--LPSRGYGVRRVLNNYLGMESKLKIC ATROPGEF7 APKSIAVPVSNY - 7 -TSY S SKOELRSSITVPISPSRFKIPHSSS---VKRV--LTAYVTKNEPRLKNLPLERSSRSSSS-----ERLSLEKCMKES

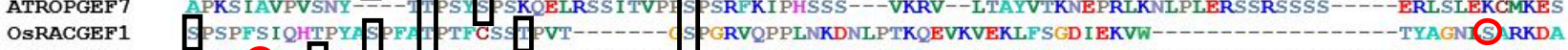

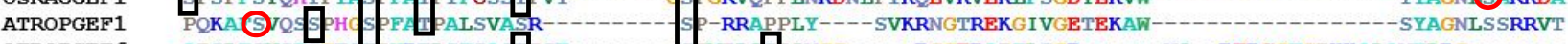

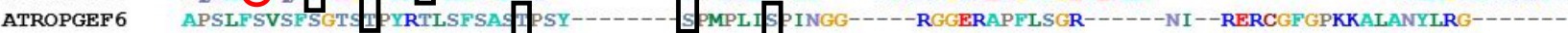

Os0590454200 VSNA-VFDLPGL----10 -

ATROPGEF5 VNPSDNADTAVINQISKDVEEEKKRNSTSVHQKGPPKYTVS

ATROPGEF7

OSRACGEF1

ATROPGEF1

ATROPGEF 6

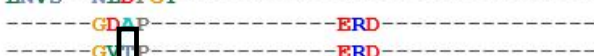




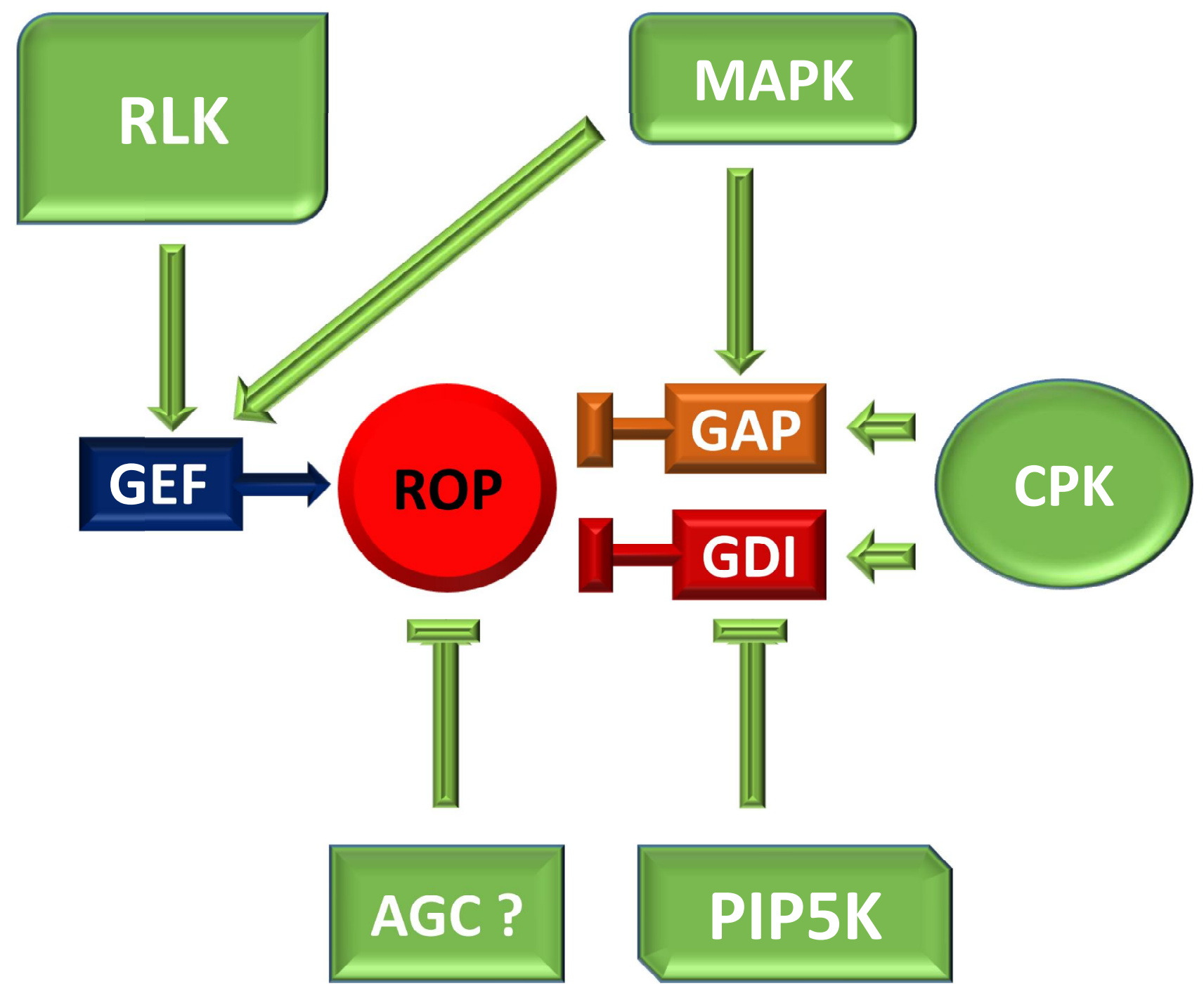




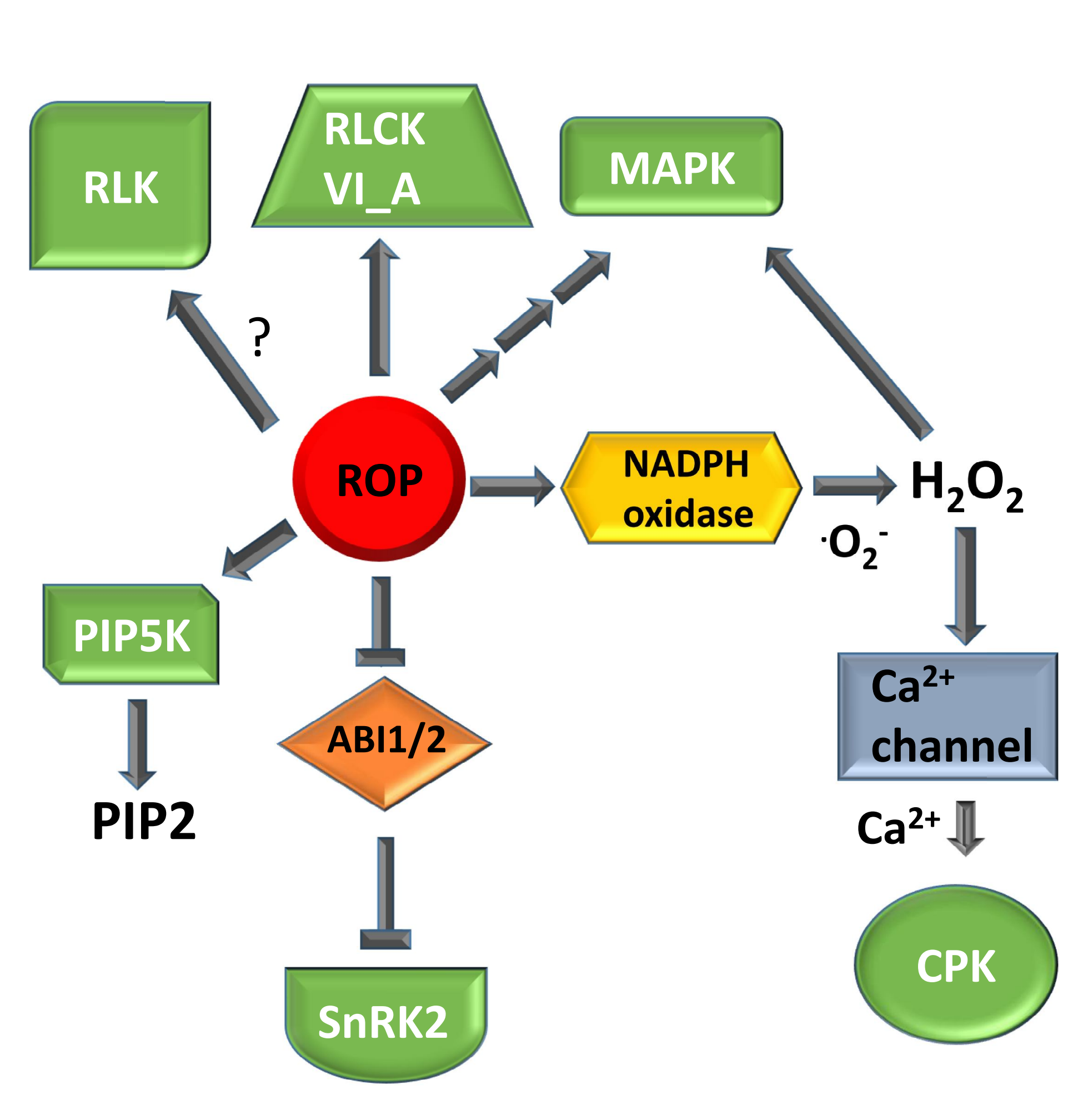

\section{Figure4 \\ Figure}

.
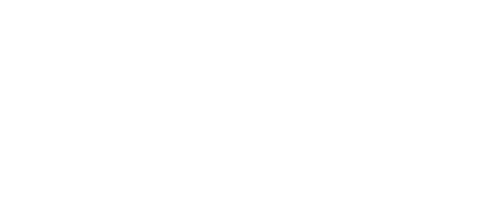

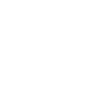

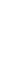

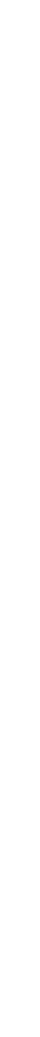

